\title{
Anti-Leishmanial Activity of Flavanone Analogues Targeting Pteridine Reductase
}

\author{
Afroze Alam ${ }^{1,2^{\star}}$, Vinay Pandit ${ }^{3}$, Shailendra Kumar ${ }^{4}$, Kamlesh Kumar Naik ${ }^{5}$, Mahfoozur Rahman, ${ }^{6}$ \\ Mohan Lal Verma ${ }^{2 *}$
}

${ }^{1}$ Narayan Institute of Pharmacy, Jamhar, Rohtas (Sasaram), Bihar, INDIA.

${ }^{2}$ Narayan Medical College and Hospital, Jamhar, Rohtas (Sasaram), Bihar, INDIA.

${ }^{3}$ Laureate Institute of Pharmacy, SH 22, VPO Kathog, Kangra, Himachal Pradesh, INDIA.

${ }^{4}$ Govt. Pharmacy Instutute, Agam kuan, Patna, Bihar, INDIA.

${ }^{5}$ Nandha College of Pharmacy, Perundurai Main Road, Erode, Tamil Nadu, INDIA.

${ }^{6}$ Department of Pharmaceutical Sciences, Faculty of Health Sciences, Sam Higginbottom Institute of Agriculture, Technology and

Sciences (SHIATS), Allahabad, Uttar Pradesh, INDIA.

\begin{abstract}
Objectives: The aim of the study is to develop new synthetic anti-leishmanial agents as flavanone analogues, which should have low toxicity with noticeable yield. Methodology: The starting materials for the synthesis of test compounds were 2'-hydroxypropiohenones, 2'-hydroxyacetophenone and substituted benzaldehyde. Test compounds were synthesized by three steps reaction starting from condensation, cyclization and reduction to yield 3 -substituted flananone analogues. The synthesized compounds were screened by in vitro anti-leishmanial assay against promastigotes of $L$. donovani. Result: A series of flavanone analogues have been synthesized using cobalt (II) phthalocyanine and $\mathrm{NaBH} 4$ in the equimolar ratio of the reactant with noticeable yield. The structures of the test compounds were elucidated and established by U.V. IR, ${ }^{1} \mathrm{H}-\mathrm{NMR},{ }^{13} \mathrm{C}-\mathrm{NMR}$ and mass spectrometry. The synthesized compounds were screened by in vitro antileishmanial assay against promastigotes of $L$. donovani. Conclusion: Most of the compounds exhibited moderate leishmanicidal activity, while some compounds such as $4 \mathrm{~b}, 10 \mathrm{~b}, 5 \mathrm{~b}$, and $3 \mathrm{a}$ have shown promising antileishmanial activity against promastigotes of $L$. donovani.
\end{abstract}

Key words: Antileishmanial activity; Cobalt (II)phthalocyanine; Flavanone derivatives; $L$. donovani; Promastigotes.

\section{INTRODUCTION}

Leishmaniasis is a vector-borne disease and considered as one of the world's most neglected diseases. The annual global incidence of leishmaniasis is approximately 12 million cases. ${ }^{1}$ Leishmaniasis is endemic in 88 countries with more than 350 million peoples are at risk. ${ }^{2,3}$ The number of cases reported globally has increased over the past 10 years due to the increase in anti-leishmanial drug resistance and lack of adequate vector or reservoir control tools. ${ }^{4}$ Antimonial compounds are the first line drugs and drug of choice for the treatment of leshmaniasis but the emergence of drug resistance against these drugs is an emerging problem and spread of drug resistance strains of Leishmania is an alarming feature. Newer drugs like Miltifosine and Amphoterisin B is approved for the treatment of leshmaniasis are highly toxic and above all are very expensive. Thus, the control of leishmaniasis has become a costly affair due to the high cost of alternative drugs. For the control of leishmaniasis, there is a need to discover a novel compound which should not only be less toxic but also cost effective. During the last few years, researchers have focused their interest on the discovery of new anti-leishman-
Submission Date: 18-09-2017; Revision Date: 08-11-2017; Accepted Date: 21-11-2017 DOI: 10.5530/ijper.52.3.56 Correspondence:

Afroze Alam, Narayan Institute of Pharmacy Jamuhar, Sasaram-821305, Bihar- INDIA. Email: afrozepharma@gmail. com

Mobile No: +918091974886 Mohan Lal Verma Narayan Medical College \& Hospital Jamuhar, Sasaram-821305, Bihar, INDIA Email: drmlvermapmch@ gmail.com

Mobile No. +918578003011

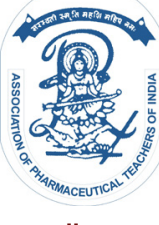

www.ijper.org 
ials by the isolation of bioactive molecules from plants, by semi synthetic and synthetic approach ${ }^{5,6}$ and some of synthetic molecules undergo clinical trials which have shown efficacy against Leishmania parasite. ${ }^{7,8,9}$

Chalcones and flavone analogues are the integral component of many pharmacological active compounds like antiprotozoal, anti-inflammatory, immunomodulatory, nitric oxide inhibition, anticancer. ${ }^{10,11}$ The molecular basis of the anti-leishmanial action is still not well established. ${ }^{12}$ The Pteridine reductase (PTR1) is a member of oxido-reductase family, which has become an attractive target for the development of novel anti-leishmanial agents. Pteridine reductase is a flavoprotein, which is unique in leishmania and plays vital role in the growth of the leishmania via generation of intermediate which is required for the synthesis of DNA precursors. ${ }^{13}$ The enzyme PTR1 is predominantly involved in reduction of biopterin to dihydrobiopterin and tetrahydrobiopterin but it is also capable to reduce dihydrofolate to tetrahydrofolate. Hence, a combined strategy to target both PTR1 and Dihydrofolate reductase (DHFR) will be more effective to stop parasitic growth and survival.

A number of other compounds have been screened against PTR1 in $L$. donovani. ${ }^{14}$ Several natural flavonoids were found to be inhibiting both DHFR and PTR1. ${ }^{15}$ So, the present strategy reflects the study of new flavanone analogues as promising anti-leishmanial agents targeting Pteridine reductase coupled with DHFR. This indicates that some novel flavanone analogues as an inhibitor are essentially required that targets both the enzymes simultaneously.

Researchers recently identified several types of flavonoids as antiprotozoal principles of plant extracts, ${ }^{16,17,18}$ but a comprehensive study of their structure-activity relationships (SARs) has not been conducted so far. Therefore, it was our fine interest to assess the in vitro leishmanicidal activities of a large 3-substituted flavanone analogues library, draw SARs, and determine their anti-leishmanial activity without significant toxicity in mammalian cells.

\section{MATERIALS AND METHODS}

\section{Chemicals}

All the chemicals and solvents used were of AR-grade and LR-grade and were obtained from Sigma-Aldrich, Sisco Research Laboratories, Qualigens, Rankem, S.D. Fine, Hi-Media and Merck.

\section{Synthesis of 3-methyl chalcone}

To a solution of 0.01 mole of substituted 2'-hydroxypropiophenones in $10 \mathrm{ml}$ of $40 \% \mathrm{KOH}$ and $20 \mathrm{ml}$ of ethyl alcohol, 0.01 mole of substituted benzaldehyde

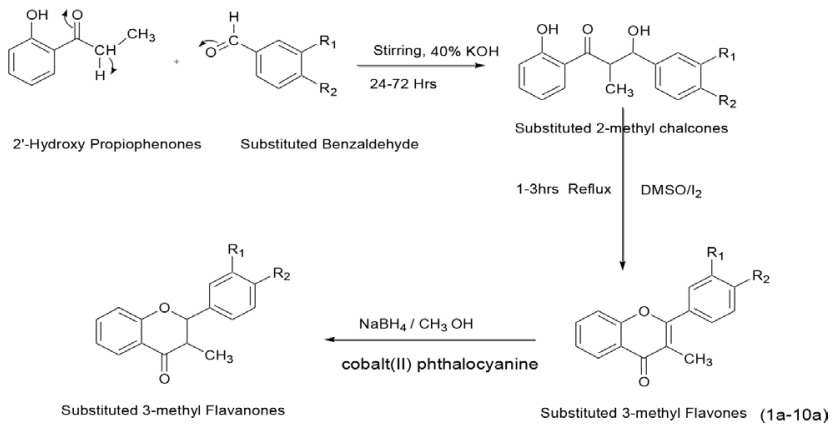

Figure 1(A): portrays the scheme-1 for synthesis of 3-methyl flavanone derivatives using various steps.

was added and mixture was stirred for 48-72 h. The coloured solution was poured into crushed ice and acidified with $1 \mathrm{~N} \mathrm{HCl}$ at $24-26^{\circ} \mathrm{C}$. The precipitate so obtained was washed with cold water, filtered, dried and recrystallized with absolute alcohol.

\section{Synthesis of 3-methyl flavone}

To a solution of 0.01 mole of chalcone in $50 \mathrm{ml}$ of dimethyl sulphoxide (DMSO) taken in $100 \mathrm{ml}$ round bottom flask fitted with reflux condenser, 15-20 granules of iodine was added. The reaction mixture was refluxed for 3-4 h and kept overnight. The precipitate was neutralized with sodium thiosulphate to remove unreacted $\mathrm{I}_{2}$ washed with water, fitted, dried and recrystallized with absolute alcohol. ${ }^{19}$

\section{Synthesis of 3-methyl flavanone}

To 0.01 moles of 3-methyl flavone in $100 \mathrm{ml}$ round bottom, the equimolar amount of $\mathrm{NaBH} 4$ and $10-15 \mathrm{ml}$ of methanol in the presence of $\mathrm{AlCl}_{3}$ was added and mixture was refluxed for 2-3 h. The resulting solution was cooled to room temperature followed by the addition of ice cold water. The solid was separated by filtration, washed with cold water and recrystallized from ethanol. The experiments suggest that the present reductive system initially reduces the conjugated double bond see Table 1..$^{20,21}$

\section{Synthesis of 2'-hydroxy chalcones}

To a solution of 0.01 mole of substituted 2'-hydroxyacetophenones in $10 \mathrm{ml}$ of $40 \% \mathrm{KOH}$ and $20 \mathrm{ml}$ of ethyl alcohol, 0.01 mole of substituted benzaldehyde was added and mixture was stirred for 48-72 h. Completion of the reaction was monitored on TLC (20\% Ethyl acetate in toluene). The coloured solution was poured into crushed ice and acidified with $1 \mathrm{~N} \mathrm{HCl}$ at $24-26^{\circ} \mathrm{C}$. The precipitate so obtained was washed with cold water, filtered, dried and recrystallized with absolute alcohol.

\section{Synthesis of 3-hydroxyflavones}


To a suspension of $0.01 \mathrm{ml}$ of chalcone, $50 \mathrm{ml}$ of ethanol was added $10 \mathrm{ml}$ of $20 \%$ aqueous sodium hydroxide with stirring, followed by the careful addition of $\mathrm{I} 5 \mathrm{ml}$ of $30 \%$ hydrogen peroxide over a period of $0.5-1 \mathrm{~h}$. The reaction mixture was stirred for $3-5 \mathrm{~h}$ at $30^{\circ} \mathrm{C}$ and completion of the reaction was monitored on TLC (20\% Ethyl acetate in toluene). After reaction completion, the mixture was poured into crushed ice containing $5 \mathrm{~N} \mathrm{HCl}$. The precipitate was filtered, washed, dried and recrystallized from ethyl acetate. ${ }^{22}$

\section{Synthesis of 3-hydroxyflavanone analogues}

To a solution of 0.01 mole of 3-hydroxyflavone, 10-15 $\mathrm{ml}$ of methanol and 0.01 mole of $\mathrm{NaBH}_{4}$ was added and the mixture was refluxed for 3-4 $\mathrm{h}$ in the presence of cobalt(II) phthalocyanine to yield 3-hydroxyflavanone analogues.

\section{Anti-leishmanial Activity}

\section{Chemicals}

Parasite stock culture: Axenic culture of L donovani (LdMIPL-1) was maintained at $25^{\circ} \mathrm{C}$ in RPMI 1640 (Himedia, India) medium supplemented with 10\% heat inactivated Fetal Bovine Serum (FBS) (Himedia, Mumbai, India), streptomycin $(150 \mu \mathrm{g} / \mathrm{mL})$, penicillin $\mathrm{G}(100 \mu \mathrm{g} / \mathrm{mL})$ and gentamycin $(150 \mu \mathrm{g} / \mathrm{mL})$ at $\mathrm{pH} 7.2$

\section{Anti-leishmanial assay}

For antileishmanial activity, pro-mastigotes of $L$ donovani were sub-cultured in Schneider's Insect Medium (Himedia, Mumbai, India) supplemented with $10 \%$ heat inactivated FBS, streptomycin $(150 \mu \mathrm{g} / \mathrm{mL})$, penicillin $\mathrm{G}(100 \mu \mathrm{g} / \mathrm{mL})$ and gentamycin $(150 \mu \mathrm{g} / \mathrm{mL})$. The antileishmanial screening was performed in 96-well flat bottom tissue culture plates (Corning Life Science, Corning USA) 100 microliters of cell suspension containing $2 \times 10^{6}$ to $3 \times 10^{6}$ cells $/ \mathrm{mL}$ was poured in each well of the plate. Four different concentrations of the methanolic extracts i.e. 100, 250, 350 and $500 \mu \mathrm{g} / \mathrm{mL}$, dissolved in dimethyl sulfoxide $(<0.025 \% \mathrm{v} / \mathrm{v})$ and added to the culture. The plates were then incubated at $25^{\circ} \mathrm{C}$ for $24 \mathrm{~h}$. Amphotericin B and sodium stibugluconate were used as positive controls and cell suspension with $0.025 \%$ DMSO was used as a negative control. Inhibition of the promastigotes was assessed by measuring the cleavage of $10 \mathrm{mg} / \mathrm{mL}$ of MTT [3-(4.5-dimethylthiazol-2-yl)-2.5-diphenyl tetrazolium bromide]. ${ }^{23,24,25}$ The absorbance was measured using an ELISA plate reader (BioTek, USA) at $595 \mathrm{~nm}$. Percent growth inhibition was calculated by the following formula:

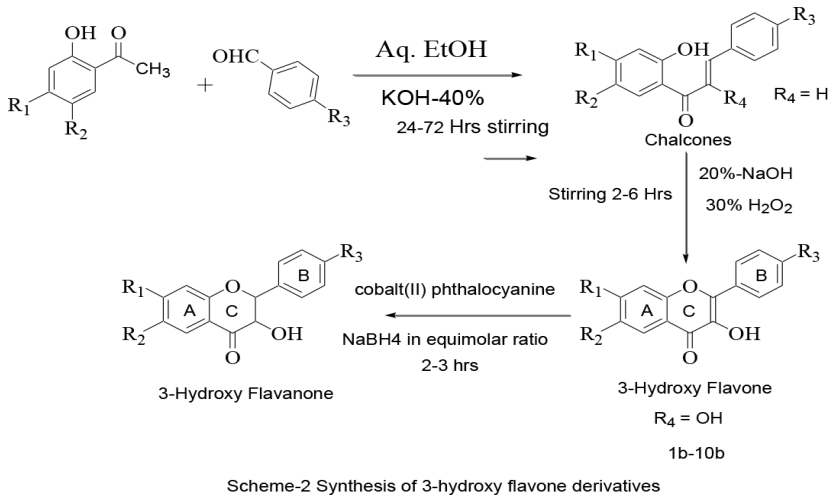

Figure 1(B): portrays the scheme-2 for synthesis of 3-hydroxy flavanone derivatives using various steps.

$$
\% \text { of Inhibition }=\frac{\text { OD Control }- \text { OD Treated }}{\text { OD Control }} \times 100
$$

Molecular docking into biopterin/dihydrofolate binding site of Pteridine reductase (PTR1).

Library of 20 substituted flavanone analogues were prepared by using chemdraw ultra 11.0. All ligands were prepared through Auto Dock Tools. The 3D crystal structure was obtained from Protein Data Bank (PDB code: 2XOX). ${ }^{26}$ The 3D structures of PTR1, (from 2XOX), was used for virtual screening. ${ }^{27}$ Docking parameters were set to default values on the basis of Lamarckian genetic algorithm principle. ${ }^{28}$ Autogrid program of AutoDock suit was used for generation of grid around binding pocket within target protein. Finally, docking simulation was carried out with AutoDock 4.2. Ligplot and UCSF Chimera version 1.8.1 were used for analysis of docking results (protein ligand interaction) and visualization of docked protein ligand complexes.

\section{RESULTS}

\section{Chemistry}

Synthesis of 3-hydroxy and 3-methylflavanone analogoues involve 3 steps (See Figure 1A and 1B). Spectral Analysis of $1 \mathrm{a}-10 \mathrm{a}$ (3.1.1.1 to 3.1.1.10) and 1b-10b (3.1.1.11 to 3.1.1.20) are available in supplementary file

\section{2-(4-fluorophenyl)-2,3,dihydro-3-methylchromen- 4-one (1a)}

Pale yellow solid, physical data is summarized in Table 2 . IR spectra $\left.(\mathrm{KBr} \mathrm{cm})^{-1}\right)$ : 3041(ArC-H), $2762(\mathrm{C}-\mathrm{H})$, $1707(\mathrm{C}=\mathrm{O}), 1514$ (aromatic $\mathrm{C}=\mathrm{C}), 1156(\mathrm{C}-\mathrm{F})$.

${ }^{1} \mathbf{H}-\mathbf{N M R} \quad(\mathbf{4 0 0 M H z}, \mathbf{C D C l} \delta, \quad$ TMS $=0): 8.2(1 \mathrm{H}$, $\mathrm{d}, J=7.72 \mathrm{~Hz}, 5-\mathrm{H}), 7.66(1 \mathrm{H}, \mathrm{dd}, J=2.76 \mathrm{~Hz}, J=7.88$ $\mathrm{Hz}, 7-\mathrm{H}), 7.65\left(2 \mathrm{H}, \mathrm{m}, 2^{\prime} 6,-\mathrm{H}\right), 7.46(1 \mathrm{H}, \mathrm{d}, J=7.84$ 
Table 1: List of various substitutions on Ring $\mathbf{A}$ and Ring B

\begin{tabular}{|c|c|c|}
\hline COMPOUNDS & $\mathbf{R}_{\mathbf{1}}$ & $\mathbf{R}_{\mathbf{2}}$ \\
\hline $\mathbf{1 a}$ & $\mathrm{H}$ & $\mathrm{F}$ \\
\hline $\mathbf{2 a}$ & $\mathrm{H}$ & $\mathrm{Cl}$ \\
\hline $\mathbf{3 a}$ & $\mathrm{H}$ & $\mathrm{OH}$ \\
\hline $\mathbf{4 a}$ & $\mathrm{H}$ & $\mathrm{OCH}_{3}$ \\
\hline $\mathbf{5 a}$ & Methylene dioxide & $\mathrm{R}_{2}$ and $\mathrm{R}_{3}$ \\
\hline $\mathbf{6 a}$ & $\mathrm{Cl}$ & $\mathrm{H}$ \\
\hline $\mathbf{7 a}$ & $\mathrm{H}$ & $\mathrm{CH}_{3}$ \\
\hline $\mathbf{8 a}$ & $\mathrm{H}$ & $\mathrm{Br}$ \\
\hline $\mathbf{9 a}$ & $\mathrm{OCH}$ & $\mathrm{H}$ \\
\hline $\mathbf{1 0 a}$ & $\mathrm{H}$ & $\mathrm{H}$ \\
\hline
\end{tabular}

$R_{1,} R_{2}$ and $R_{3}$ are the different substitution of ring- $B$

$\mathrm{Hz}, 6-\mathrm{H}), 7.40(1 \mathrm{H}, \mathrm{d}, \mathrm{J}=7.32 \mathrm{~Hz}, 8-\mathrm{H}), 7.05(2 \mathrm{H}, \mathrm{m}$, 5',3'-H), $4.50(1 \mathrm{H}, \mathrm{d}, 3-\mathrm{H} \mathrm{J}=7.01 \mathrm{~Hz}), 5.12(1 \mathrm{H}, \mathrm{d}$, $2-\mathrm{H} J=12.36 \mathrm{~Hz}), 2.19\left(3 \mathrm{H}\right.$, s, $\left.3-\mathrm{CH}_{3}\right) \cdot,{ }^{13} \mathbf{C} \mathbf{~ N M R}$ $(400 \mathrm{MHz}, \delta, \mathrm{CDCl} 3, \mathrm{TMS}=0)$ : Aromatic RingA $(156.19,135.24,131.07,130.03,125.94,118.76$, $182.88(\mathrm{C}=\mathrm{O}), 125.36$ (2-C), 109.78 (3-C), Aromatic Ring B (159.47, 128.45, 128.03, 116.68, 115.50,) 76.58 (5-C), 42.45(4-C), 25-3CH $\mathrm{CH}_{3}$. TOF MS ES+ m/z) 257

\section{2-(4-chlorophenyl)-2,3, dihydro-3-methylchromen- 4-one(2a)}

Light yellow solid, the physical data is summarized in Table 2.

IR Spectra $\left(\mathrm{KBr} \mathrm{cm}^{-1}\right): 3052(\mathrm{ArC}-\mathrm{H}), 2951(\mathrm{C}-\mathrm{H})$, $1700(\mathrm{C}=\mathrm{O}), 1495$ (aromatic $\mathrm{C}=\mathrm{C}), 758(\mathrm{C}-\mathrm{Cl})$. ${ }^{1} \mathrm{H}-\mathrm{NMR}\left(400 \mathrm{MHz}, \mathbf{C D C l}_{3} \delta\right.$, TMS $\left.=0\right): 8.26(1 \mathrm{H}, \mathrm{d}$, $J=8.62 \mathrm{~Hz}, 5-\mathrm{H}), 8.23(1 \mathrm{H}, \mathrm{d}, J=8.62 \mathrm{~Hz}, 7-\mathrm{H}), 7.74(2 \mathrm{H}$, m,2',6'-H), $7.60(1 \mathrm{H}, \mathrm{dd}, J=8.52 \mathrm{~Hz}, 6-\mathrm{H}), 7.52(1 \mathrm{H}$, $\mathrm{d}, \mathrm{J}=8.44 \mathrm{~Hz}, 8-\mathrm{H}), 7.54\left(2 \mathrm{H}, \mathrm{m}, 5^{\prime}, 3^{\prime}-\mathrm{H}\right), 4.55(1 \mathrm{H}, \mathrm{d}$, $3-\mathrm{H} \mathrm{J}=7.01 \mathrm{~Hz}), 5.23(1 \mathrm{H}, \mathrm{d}, 2-\mathrm{H} J=12.36 \mathrm{~Hz}), 2.1(3 \mathrm{H}$, s, $\left.2-\mathrm{CH}_{3}\right),{ }^{13} \mathrm{C}$ NMR (400 MHz, $\delta, \mathbf{C D C l} 3, \mathbf{T M S}=0$ ): Aromatic Ring-A (157.19, 135.04, 132. 07, 129.03, 123.94, 116.16,) $181.88(\mathrm{C}=\mathrm{O}), 126.36$ (2-C), 113.78 (3-C), Aromatic Ring B (139.47, 128.45, 128.03, 127.13, $126.68,125.50$,) $20-3 \mathrm{CH}_{3}$. TOF MS ES+ m/z) 272.5

\section{2,3-dihydro-2-(4-hydroxypenyl)-3- methylchromen-4-one (3a)}

Dark brown solid, physical data is summarized in Table 2.

IR Spectra $\left.(\mathrm{KBr} \mathrm{cm})^{-1}\right): 3570(\mathrm{Ar}-\mathrm{OH}), 3060$ (ArC$\mathrm{H}), 2870(\mathrm{C}-\mathrm{H}), 1715(\mathrm{C}=\mathrm{O}), 1512$ (aromatic $\mathrm{C}=\mathrm{C})$, ${ }^{1} \mathbf{H}-\mathbf{N M R}(\mathbf{4 0 0 M H z}, \mathbf{C D C l} \delta$, TMS $=0): 8.12(1 \mathrm{H}, \mathrm{d}$, $J=7.75 \mathrm{~Hz}, 5-\mathrm{H}), 7.60(1 \mathrm{H}, \mathrm{d}, J=7.52 \mathrm{~Hz}, 7-\mathrm{H}), 7.50$ $\left(2 \mathrm{H}, \mathrm{m}, 6^{\prime}, 2^{\prime}-\mathrm{H}\right), 7.21(1 \mathrm{H}, \mathrm{dd}, J=7.90 \mathrm{~Hz}, 6-\mathrm{H}), 6.99$ $\left(2 \mathrm{H}, \mathrm{m}, 5^{\prime}, 3^{\prime}-\mathrm{H}\right), 6.80(1 \mathrm{H}, \mathrm{d}, \mathrm{J}=7.75 \mathrm{~Hz}, 8-\mathrm{H}), 4.87$ $(1 \mathrm{H}, \mathrm{d}, 3-\mathrm{H} \mathrm{J}=7.67 \mathrm{~Hz}), 5.45(1 \mathrm{H}, \mathrm{d}, 2-\mathrm{H} J=12.36 \mathrm{~Hz})$, $1.9\left(3 \mathrm{H}, \mathrm{s}, 2-\mathrm{CH}_{3}\right) \cdot{ }^{13} \mathrm{C}$ NMR $(400 \mathrm{MHz}, \delta, \mathbf{C D C l} 3$, TMS=0): Aromatic Ring-A (156.19, 135.24, 131. 24, 130.03, 122.94, 115.06, $183.88(\mathrm{C}=\mathrm{O}), 125.36(2-\mathrm{C})$, 115.78 (3-C), Aromatic Ring B (152.47, 126.45, 125.03, $123.44,117.13,116.68,) 10-3 \mathrm{CH}_{3}$. TOF MS ES+ m/z) 255.5

\section{2,3-dihydro-2-(3-hydroxy-4-methoxypenyl)-3-} methylchromen-4-one (4a)

yellow solid, physical data is summarized in Table 2.

IR spectra $\left(\mathrm{KBr} \mathrm{cm}^{-1}\right)$ : 3074( $\left.\mathrm{ArC}-\mathrm{H}\right), 2851(\mathrm{C}-\mathrm{H}), 1685$ $(\mathrm{C}=\mathrm{O}), 1514$ (aromatic C=C), $1182(\mathrm{C}-\mathrm{O}) .{ }^{1} \mathbf{H}-\mathbf{N M R}$ $\left(400 \mathrm{MHz}, \mathrm{CDCl}_{3} \delta\right.$, TMS $\left.=0\right): 7.94(1 \mathrm{H}, \mathrm{d}, J=9.56 \mathrm{~Hz}$, $5-\mathrm{H}), 7.51(1 \mathrm{H}, \mathrm{dd}, \mathrm{J}=8.64 \mathrm{~Hz}, 2.8 \mathrm{~Hz}, 7-\mathrm{H}), 7.47(1 \mathrm{H}$, $\mathrm{dd}, \mathrm{J}=7.45 \mathrm{~Hz}, \mathrm{~J}=2.8 \mathrm{~Hz}, 6-\mathrm{H}), 7.07(1 \mathrm{H}, \mathrm{d}, \mathrm{J}=8.2 \mathrm{~Hz}$, $8-\mathrm{H}), 7.02\left(1 \mathrm{H}, \mathrm{d}, \mathrm{J}=8.45 \mathrm{~Hz}, 6{ }^{\prime}-\mathrm{H}\right), 6.98(1 \mathrm{H}, \mathrm{d}$, $\left.\mathrm{J}=1.96 \mathrm{~Hz}, 2^{\prime}-\mathrm{H}\right), 6.91\left(1 \mathrm{H}, \mathrm{d}, \mathrm{J}=7.88 \mathrm{~Hz}, 5^{\prime} \mathrm{H}\right), 5.02(1 \mathrm{H}, \mathrm{s}$, 3'-OH) Exchangeable with $\mathrm{D}_{2} \mathrm{O}, 4.30(1 \mathrm{H}, \mathrm{d}, 3-\mathrm{H}$ $\mathrm{J}=7.67 \mathrm{~Hz}), 5.56(1 \mathrm{H}, \mathrm{d}, 2-\mathrm{H} J=12.36 \mathrm{~Hz}), \quad 3.92(3 \mathrm{H}$, s, 4'- $\left.\mathrm{OCH}_{3}\right), 2.19\left(3 \mathrm{H}, \mathrm{s}, 3-\mathrm{CH}_{3}\right) \cdot{ }^{13} \mathbf{C}$ NMR (400 MHz, $\boldsymbol{\delta}, \mathbf{C D C 1 3}, \mathbf{T M S}=\mathbf{0})$ : Aromatic Ring-A $(156.29,135.24$, 132.24, 129.03, 123.94, 116.06,) 182.88 (C=O), 145.36 (2-C), 112.78 (3-C), Aromatic Ring B (155.47, 128.45, 128.03, 124.34, 115.13, 116.68,), 60.5 -OCH3, (10.09-3 $\mathrm{CH}_{3}$ ), TOF MS ES+ m/z) 287.5

2-(benzo[d][1,3]dioxal-6-yl)-2,3-dihydro-3methylchromene-4-one(5a)

Yellow solid, physical data is summarized in Table 2. IR Spectra $\left(\mathrm{KBr} \mathrm{cm}^{-1}\right)$ : 3074 (ArC-H), $2887(\mathrm{C}-\mathrm{H}), 1682$ $(\mathrm{C}=\mathrm{O}), 1490$ (aromatic $\mathrm{C}=\mathrm{C}), 1249(\mathrm{C}-\mathrm{O}) .{ }^{1} \mathbf{H}-\mathbf{N M R}$ (400MHz, $\mathbf{C D C l}_{3} \delta$, TMS $\left.=0\right): 8.23(1 \mathrm{H}, \mathrm{d}, J=8.32 \mathrm{~Hz}$, $5-\mathrm{H}), 7.69(1 \mathrm{H}, \mathrm{d}, J=7.08 \mathrm{~Hz}, 7-\mathrm{H}), 7.56(1 \mathrm{H}, \mathrm{dd}, J=8.4$ $\mathrm{Hz}, J=8.53 \mathrm{~Hz}, 6-\mathrm{H}), 7.42(1 \mathrm{H}, \mathrm{d}, J=6.89 \mathrm{~Hz} .8-\mathrm{H})$, $7.38\left(1 \mathrm{H}, \mathrm{d}, \mathrm{J}=7.00 \mathrm{~Hz} .2^{\prime}-\mathrm{H}\right), 6.96(1 \mathrm{H}, \mathrm{d}, J=8.24 \mathrm{~Hz}$, 5'-H), 6.71( (1H,d, J= 7.56Hz,6'-H), 6.0(2H, s, 4"'-CH2). $4.40(1 \mathrm{H}, \mathrm{d}, 3-\mathrm{H} \mathrm{J}=7.80 \mathrm{~Hz}), 5.60(1 \mathrm{H}, \mathrm{d}, 2-\mathrm{H}$ $J=12.56 \mathrm{~Hz}), 1.8\left(3 \mathrm{H}, \mathrm{s}, 3-\mathrm{CH}_{3}\right) \cdot{ }^{13} \mathbf{C}$ NMR (400 MHz, $\delta$, CDC13, TMS=0): Aromatic Ring-A (157.29, 133.24, 132.24, 128.03, 122.94, 115.06,) 183.88 (C=O), 155.36 (2-C), 110.78 (3-C), Aromatic Ring B (149.47, 148.45, 126.03, 120.34, 116.13, 112.68, 107,23), (9.09-3 $\left.\mathrm{CH}_{3}\right)$, TOF MS ES+ m/z) 283.5

2-(2-chlorophenyl)-2,3, dihydro-3-methylchromen4-one (6a): Light yellow solid, the physical data is summarized in Table 2.

IR Spectra $\left(\mathrm{KBr} \mathrm{cm}^{-1}\right)$ : $3050(\mathrm{ArC}-\mathrm{H}), 2805(\mathrm{C}-\mathrm{H})$, $1645(\mathrm{C}=\mathrm{O}), 1510$ (aromatic $\mathrm{C}=\mathrm{C}), 745(\mathrm{C}-\mathrm{Cl}) .{ }^{1} \mathrm{H}-$ NMR (400MHz, $\mathbf{C D C l}_{3} \delta$, TMS $\left.=0\right): 8.2(1 \mathrm{H}, \mathrm{d}, J=8.64$ $\mathrm{Hz}, 5-\mathrm{H}), 7.8(1 \mathrm{H}, \mathrm{d}, J=8.24 \mathrm{~Hz}, 7-\mathrm{H}), 7.44(1 \mathrm{H}, \mathrm{d}, J=7.6$ $\left.\mathrm{Hz}, 3^{\prime}-\mathrm{H}\right), 7.23\left(1 \mathrm{H}, \mathrm{d} J=7.96 \mathrm{~Hz}, 6{ }^{\prime}-\mathrm{H}\right), 7.01$ (1H, dd, $\left.J=2.2 \mathrm{~Hz}, J=7.8 \mathrm{~Hz}, 4^{\prime}-\mathrm{H}\right), 6.92(1 \mathrm{H}, \mathrm{dd}, J=2.0 \mathrm{~Hz}$, 
Table 2: Physicochemical characterization of 3-methylflavanone analogues

\begin{tabular}{|c|c|c|c|c|c|c|c|c|c|c|c|}
\hline \multirow[t]{2}{*}{ Code } & \multirow{2}{*}{$\begin{array}{c}\text { Molecular } \\
\text { Formula }\end{array}$} & \multirow{2}{*}{$\begin{array}{l}\text { Molecular } \\
\text { Weight }\end{array}$} & \multirow{2}{*}{$\begin{array}{l}(\%) \\
\text { Yield }\end{array}$} & \multirow{2}{*}{$\begin{array}{c}\text { Melting } \\
\text { Point }\left({ }^{\circ} \mathrm{C}\right)\end{array}$} & \multirow{2}{*}{$\begin{array}{c}\lambda_{\max } \\
\mathrm{nm}\end{array}$} & \multirow{2}{*}{$\begin{array}{c}\mathbf{R}_{\mathrm{f}} \\
\text { Value }\end{array}$} & \multicolumn{5}{|c|}{ Elemental Analysis Calculated (\%) } \\
\hline & & & & & & & C & $\mathbf{H}$ & 0 & $\mathbf{N}$ & $\mathrm{Cl} / \mathrm{F}$ \\
\hline $1 a$ & $\mathrm{C}_{16} \mathrm{H}_{13} \mathrm{FO}_{2}$ & 256.02 & 73 & $95-100$ & 341 & 0.73 & 77.20 & 5.27 & 13.06 & & 7.90 \\
\hline $2 a$ & $\mathrm{C}_{16} \mathrm{H}_{13} \mathrm{ClO}_{2}$ & 272.71 & 80 & $170-175$ & 351 & 0.62 & 69.67 & 4.93 & 12.60 & - & 12.00 \\
\hline $3 a$ & $\mathrm{C}_{16} \mathrm{H}_{14} \mathrm{O}_{3}$ & 254.08 & 82 & $125-130$ & 348 & 0.71 & 77.42 & 5.23 & 20.04 & - & - \\
\hline $4 a$ & $\mathrm{C}_{17} \mathrm{H}_{16} \mathrm{O}_{3}$ & 268.29 & 90 & $90-95$ & 343 & 0.57 & 77.44 & 5.50 & 19.56 & - & - \\
\hline $5 a$ & $\mathrm{C}_{17} \mathrm{H}_{14} \mathrm{O}_{4}$ & 282.27 & 70 & $110-115$ & 308 & 0.65 & 73.48 & 5.66 & 21.14 & - & - \\
\hline $6 a$ & $\mathrm{C}_{16} \mathrm{H}_{13} \mathrm{ClO}_{2}$ & 272.04 & 80 & $165-170$ & 328 & 0.56 & 71.12 & 5.00 & 12.98 & - & 13.90 \\
\hline $7 a$ & $\mathrm{C}_{17} \mathrm{H}_{16} \mathrm{O}_{2}$ & 252.29 & 90 & $102-105$ & 336 & 0.58 & 82.20 & $6 . .10$ & 13.25 & & - \\
\hline $8 a$ & $\mathrm{C}_{16} \mathrm{H}_{13} \mathrm{BrO}_{2}$ & 315.99 & 70 & 173-177 & 344 & 0.87 & 61.03 & 4.57 & 11.24 & - & $26.19(\mathrm{Br})$ \\
\hline $9 a$ & $\mathrm{C}_{17} \mathrm{H}_{16} \mathrm{O}_{3}$ & 268.29 & 90 & $110-114$ & 345 & 0.55 & 77.44 & 5.50 & 19.56 & - & - \\
\hline $10 a$ & $\mathrm{C}_{16} \mathrm{H}_{12} \mathrm{O}_{2}$ & 238.27 & 80 & $98-112$ & 310 & 0.66 & 80.12 & 5.94 & 14.25 & - & - \\
\hline
\end{tabular}

Physical Characterizations of 3-methylflavanone derivatives:. (TLC Solvent Used: Tolvene : Ethyl Acetate 8:2)

$\%$ yields range between $70-90$ and other physical parameter were determined by usual methods.

$\left.J=7.32 \mathrm{~Hz}, 5^{\prime}-\mathrm{H}\right), 6.90(1 \mathrm{H}, \mathrm{d}, \mathrm{J}=7.89 \mathrm{~Hz}, 6-\mathrm{H}), 6.88(1 \mathrm{H}$, $\mathrm{d}, \mathrm{J}=7.50 \mathrm{~Hz}, 8-\mathrm{H}), 4.50(1 \mathrm{H}, \mathrm{d}, 3-\mathrm{H} \mathrm{J}=7.80 \mathrm{~Hz}), 5.65$ $(1 \mathrm{H}, \mathrm{d}, 2-\mathrm{H} J=12.56 \mathrm{~Hz}), 1.9\left(3 \mathrm{H}, \mathrm{s}, 2-\mathrm{CH}_{3}\right) \cdot{ }^{13} \mathbf{C} \mathbf{~ N M R}$ (400 MHz, $\delta, \mathbf{C D C 1 3}$, TMS=0): Aromatic Ring-A $(156.29,132.24,131.24,129.03,123.94,116.16)$, $(\mathrm{C}=\mathrm{O}), 158.26$ (2-C), 112.43 (3-C), Aromatic Ring B (136.23, 132.56., 131.89, 129.34, 126.13, 124.68,), (8.23 $\left.-3 \mathrm{CH}_{3}\right)$, TOF MS ES+ m/z) 273.5

2,3-dihydro-3-methyl-2-p-tolylchromen-4-one (7a): white yellow solid, the physical data is summarized in Table 2. IR spectra $\left(\mathrm{KBr} \mathrm{cm}^{-1}\right): 3080(\mathrm{ArC}-\mathrm{H}), 2910$ $(\mathrm{C}-\mathrm{H}), 1690(\mathrm{C}=\mathrm{O}), 1520$ (aromatic $\mathrm{C}=\mathrm{C}), 1182(\mathrm{C}-\mathrm{O})$. ${ }^{1} \mathrm{H}-\mathrm{NMR}\left(\mathbf{4 0 0 M H z}, \mathbf{C D C l}_{3} \delta\right.$, TMS $\left.=0\right): 8.21(1 \mathrm{H}, \mathrm{d}$, $J=7.72 \mathrm{~Hz}, 5-\mathrm{H}), 7.98(1 \mathrm{H}, \mathrm{dd}, J=8.64 \mathrm{~Hz}, 7.04 \mathrm{~Hz}, 7-$ H),7.82 (2H, m, 2',6'-H), 7.75 (2H, m, 3', 5', -H ), 7.38 $(1 \mathrm{H}, \mathrm{d}, \mathrm{J}=7.2 \mathrm{~Hz}, 6-\mathrm{H}), 7.05(1 \mathrm{H}, \mathrm{d}, \mathrm{J}=7.8 \mathrm{~Hz}, 8-\mathrm{H}), 4.50$ $(1 \mathrm{H}, \mathrm{d}, 3-\mathrm{H} \mathrm{J}=7.80 \mathrm{~Hz}), 5.65(1 \mathrm{H}, \mathrm{d}, 2-\mathrm{H} J=12.56 \mathrm{~Hz})$, $2.06\left(3 \mathrm{H}, \mathrm{s}, 4^{\prime} \mathrm{CH}_{3}\right)$ 1.9. $\left(3 \mathrm{H}, \mathrm{s}, 3-\mathrm{CH}_{3}\right) \cdot{ }^{13} \mathbf{C}$ NMR (400 MHz, $\boldsymbol{\delta}, \mathbf{C D C 1 3}$, TMS $=0$ ): Aromatic Ring-A (157.29, 135.26, 133.24, 130.03, 125.94, 118.24,) 183.88 (C=O), 159.26 (2-C), 111.43 (3-C), Aromatic Ring B (138.23, 130.56., 129.89, 127.34, 126.13, 125.68,), $\left(30.67-4^{\prime} \mathrm{CH}_{3}\right)$ $\left(10.33-3 \mathrm{CH}_{3}\right)$, TOF MS ES+ m/z) 253.1.1.

\section{2-(2-bromophenyl)-2,3,dihydro-3-methylchromen- 4-one(6a)}

Light yellow solid, the physical data is summarized in Table 2.

8a: IR spectra $\left(\mathrm{KBr} \mathrm{cm}^{-1}\right)$ : $3072(\mathrm{ArC}-\mathrm{H}), 2875(\mathrm{C}-\mathrm{H})$, 1663 (C=O), 1525 (Ar C=C), 1182 (C-O). 612 (C-Br). ${ }^{1} \mathbf{H}-N M R\left(400 \mathrm{MHz}, \mathbf{C D C l}_{3} \delta\right.$, TMS=0): 7.92(1H, d, $J=7.70 \mathrm{~Hz}, 5-\mathrm{H}), 7.82(1 \mathrm{H}, \mathrm{dd}, J=8.04 \mathrm{~Hz}, 7-\mathrm{H}), 7.82$ $\left(2 \mathrm{H}, \mathrm{m}, 2^{\prime}, 6^{\prime}-\mathrm{H}\right), 7.75\left(2 \mathrm{H}, \mathrm{m}, 3^{\prime}, 5^{\prime},-\mathrm{H}\right), 7.38$ (1H,d,
$\mathrm{J}=7.2 \mathrm{~Hz}, 6-\mathrm{H}), \quad 7.05(1 \mathrm{H}, \mathrm{d}, \mathrm{J}=7.8 \mathrm{~Hz}, 8-\mathrm{H}), 4.50$ $(1 \mathrm{H}, \mathrm{d}, 3-\mathrm{H} \mathrm{J}=7.80 \mathrm{~Hz}), 5.65(1 \mathrm{H}, \mathrm{d}, 2-\mathrm{H} J=12.56 \mathrm{~Hz})$, $2.00\left(3 \mathrm{H}, \mathrm{s}, 3-\mathrm{CH}_{3}\right) \cdot{ }^{13} \mathrm{C}$ NMR $(400 \mathrm{MHz}, \delta, \mathbf{C D C l} 3$, TMS=0): Aromatic Ring-A (156.29, 134.35, 132.79, 129.03, 125.94, 118.24, $182.88(\mathrm{C}=\mathrm{O}), 155.26(2-\mathrm{C})$, 110.43 (3-C), Aromatic Ring B (130.83, 130.56., 129.29, 127.24, 127.13, 123.08,), (12.33-3 $\left.\mathrm{CH}_{3}\right)$,. TOF MS ES+ $\mathrm{m} / \mathrm{z}) 317.5$.

\section{2,3-dihydro-2-(4-methoxypenyl)-3- methylchromen-4-one (9a)}

Yellow-white solid, physical data is summarized in Table 2. IR spectra $\left(\mathrm{KBr} \mathrm{cm}^{-1}\right): 3072(\mathrm{ArC}-\mathrm{H}), 2850(\mathrm{C}-\mathrm{H})$, $16890(\mathrm{C}=\mathrm{O}), 1524$ (Ar C=C), $1180(\mathrm{C}-\mathrm{O}) .{ }^{1} \mathbf{H}-\mathbf{N M R}$ (400MHz, $\mathbf{C D C l}_{3} \delta$, TMS $\left.=0\right): 8.32(1 \mathrm{H}, \mathrm{d}, J=8.32 \mathrm{~Hz}$, $5-\mathrm{H}), 7.65(1 \mathrm{H}, \mathrm{dd}, \mathrm{J}=7.08 \mathrm{~Hz}, \mathrm{~J}=2.1 \mathrm{~Hz}, 7-\mathrm{H}), 7.56$ $\left(2 \mathrm{H}, \mathrm{m}, 2^{\prime}, 6^{\prime}-\mathrm{H}\right), 7.44\left(2 \mathrm{H}, \mathrm{m}, 3^{\prime}, 5^{\prime}-\mathrm{H}\right), 6.96(1 \mathrm{H}, \mathrm{d}$, $\mathrm{J}=7.04 \mathrm{~Hz}, 6-\mathrm{H}), 6.71(1 \mathrm{H}, \mathrm{d}, \mathrm{J}=7.8 \mathrm{~Hz}, 8-\mathrm{H}), 4.50$ $(1 \mathrm{H}, \mathrm{d}, 3-\mathrm{H} \mathrm{J}=7.80 \mathrm{~Hz}), 5.65(1 \mathrm{H}, \mathrm{d}, 2-\mathrm{H} J=12.56 \mathrm{~Hz})$, $3.92\left(3 \mathrm{H}, \mathrm{s}, 4^{\prime}-\mathrm{OCH}_{3}\right), 2.1\left(3 \mathrm{H}, \mathrm{s}, 3-\mathrm{CH}_{3}\right) \cdot{ }^{13} \mathbf{C}$ NMR (400 MHz, $\boldsymbol{\delta}, \mathbf{C D C l} 3, \mathbf{T M S}=\mathbf{0})$ : Aromatic Ring-A (156.29, 135.24, 132.24, 129.03, 123.94, 116.06, $182.88(\mathrm{C}=\mathrm{O})$, 145.36 (2-C), 112.78 (3-C), Aromatic Ring B (159.47, 131.45, 129.03, 118.34, 115.13, 110.68,), 59.5-OCH3, (9.09-3 $\left.\mathrm{CH}_{3}\right)$, TOF MS ES+ m/z) 269.5

\section{2,3-dihydro--3-methyl-2-phenylchromen-4-one (10a)}

Yellow solid, physical data is summarized in Table 2. IR spectra $\left(\mathrm{KBr}, \mathrm{cm}^{-1}\right)$ : 3085( $\left.\mathrm{ArC}-\mathrm{H}\right), 2920(\mathrm{C}-\mathrm{H})$, $1690(\mathrm{C}=\mathrm{O}), 1520$ ( $\mathrm{ArC}=\mathrm{C}), 1182(\mathrm{C}-\mathrm{O}) .{ }^{1} \mathbf{H}-\mathbf{N M R}$ (400MHz, $\mathbf{C D C l}_{3} \delta$, TMS =0): $7.20(1 \mathrm{H}, \mathrm{d}, J=7.70 \mathrm{~Hz}, 5-\mathrm{H})$, $7.09(1 \mathrm{H}, \mathrm{dd}, \mathrm{J}=8.60 \mathrm{~Hz}, 7.04 \mathrm{~Hz}, 7-\mathrm{H}), 7.00\left(2 \mathrm{H}, \mathrm{m}, 2^{\prime}, 6^{\prime}-\mathrm{H}\right)$, $6.98\left(2 \mathrm{H}, \mathrm{m}, 3^{\prime}, 5\right.$ ', - -H $), 6.90\left(1 \mathrm{H}, \mathrm{d}, \mathrm{J}=7.80 \mathrm{~Hz}, 4^{\prime}-\mathrm{H}\right)$, 


\begin{tabular}{|c|c|c|c|c|c|c|c|c|c|c|c|}
\hline & & $\begin{array}{l}\text { chem } \\
\text { Phys }\end{array}$ & are & ation $c$ & & & $\begin{array}{l}e \text { a } \\
\text { flas }\end{array}$ & ue & $\begin{array}{l}\text { 10b) } \\
\text { jues }\end{array}$ & & \\
\hline \multirow{2}{*}{$\begin{array}{l}\text { Com. } \\
\text { Code }\end{array}$} & \multirow{2}{*}{$\begin{array}{l}\text { Molecular } \\
\text { Formula }\end{array}$} & \multirow{2}{*}{$\begin{array}{l}\text { Molecular } \\
\text { Weight }\end{array}$} & \multirow{2}{*}{$\begin{array}{l}(\%) \\
\text { Yield }\end{array}$} & \multirow{2}{*}{$\begin{array}{l}\text { Melting } \\
\text { Point }\left({ }^{\circ} \mathrm{C}\right)\end{array}$} & \multirow{2}{*}{$\begin{array}{l}\chi_{\max } \\
\mathrm{nm}\end{array}$} & \multirow{2}{*}{$\begin{array}{c}\mathbf{R}_{\mathrm{f}} \\
\text { Value }\end{array}$} & \multicolumn{5}{|c|}{ Elemental Analysis Calculated (\%) } \\
\hline & & & & & & & C & $\mathbf{H}$ & 0 & $\mathbf{N}$ & $\mathrm{Cl}$ \\
\hline $1 b$ & $\mathrm{C}_{17} \mathrm{H}_{17} \mathrm{NO}_{3}$ & 283.32 & 88.24 & $134-136$ & 341 & 0.63 & 72.58 & 5.37 & 17.06 & 4.98 & - \\
\hline $2 b$ & $\mathrm{C}_{15} \mathrm{H}_{11} \mathrm{ClO}_{3}$ & 274.68 & 88.23 & $105-107$ & 351 & 0.48 & 696.07 & 3.33 & 17.60 & - & 13.00 \\
\hline $3 b$ & $\mathrm{C}_{13} \mathrm{H}_{10} \mathrm{O}_{4}$ & 230.20 & 91.00 & $110-112$ & 348 & 0.59 & 68.42 & 3.53 & 28.04 & - & - \\
\hline $4 \mathrm{~b}$ & $\mathrm{C}_{18} \mathrm{H}_{19} \mathrm{NO}_{4}$ & 313.33 & 86.00 & $168-170$ & 343 & 0.56 & 69.44 & 5.50 & 20.56 & 4.50 & - \\
\hline $5 b$ & $\mathrm{C}_{16} \mathrm{H}_{13} \mathrm{ClO}_{4}$ & 304.70 & 83.00 & 107-109 & 308 & 0.44 & 63.48 & 3.66 & 21.14 & - & 11.71 \\
\hline $6 \mathrm{~b}$ & $\mathrm{C}_{14} \mathrm{H}_{12} \mathrm{O}_{5}$ & 260.22 & 90.32 & $120-122$ & 328 & 0.50 & 65.12 & 3.90 & 30.98 & - & - \\
\hline $7 b$ & $\mathrm{C}_{18} \mathrm{H}_{19} \mathrm{NO}_{3}$ & 297.33 & 79.00 & $180-182$ & 336 & 0.57 & 73.20 & $5 . .80$ & 16.25 & 4.74 & - \\
\hline $8 \mathrm{~b}$ & $\mathrm{C}_{16} \mathrm{H}_{13} \mathrm{ClO}_{3}$ & 288.70 & 70.00 & $112-114$ & 344 & 0.42 & 67.03 & 3.87 & 16.74 & - & 12.37 \\
\hline $9 \mathrm{~b}$ & $\mathrm{C}_{14} \mathrm{H}_{12} \mathrm{O}_{4}$ & 244.22 & 79.00 & $147-149$ & 345 & 0.49 & 69.42 & 4.16 & 26.42 & - & - \\
\hline $10 \mathrm{~b}$ & $\mathrm{C}_{15} \mathrm{H}_{11} \mathrm{NO}_{5}$ & 285.05 & 85 & $135-139$ & 310 & 0.67 & 64.12 & 4.14 & 29.25 & 5.28 & - \\
\hline
\end{tabular}

(TLC Solvent Used:Tolvene : Ethyl Acetate 8:2); \%Yield ranging between 70-90\% and other physical parameter were determined by usual methods.

The antileishmanial activity of synthesized compounds were screened against promastigotes of L.donovani. Amphotericin B and Sodium Stibugluconate were used as standard drugs and exhibited $100 \%$ inhibition.

$6.88(1 \mathrm{H}, \mathrm{d}, \mathrm{J}=7.21 \mathrm{~Hz}, 6-\mathrm{H}), \quad 6.75(1 \mathrm{H}, \mathrm{d}, \mathrm{J}=7.82 \mathrm{~Hz}$, $8-\mathrm{H}), 4.50(1 \mathrm{H}, \mathrm{d}, 3-\mathrm{H} \mathrm{J}=7.80 \mathrm{~Hz}), 5.65(1 \mathrm{H}, \mathrm{d}, 2-\mathrm{H}$ $\left.J=12.56 \mathrm{~Hz}), 1.98\left(3 \mathrm{H}, \mathrm{s}, 3-\mathrm{CH}_{3}\right) \cdot\right) \cdot{ }^{13} \mathbf{C}$ NMR $(400 \mathrm{MHz}$, $\delta, \mathbf{C D C l}$, TMS $=\mathbf{0})$ : Aromatic Ring-A (159.29, 134.24, 132.27, 129.83, 122.94, 117.06,) 183.88 (C=O), 157.36 (2-C), 110.78 (3-C), Aromatic Ring B (160.47, 130.45, 130.03, 128.34, 128.13, 127.68,), (9.09-3 $\left.\mathrm{CH}_{3}\right)$,. TOF MS ES+ m/z) 239.5

\section{2-(4-(dimethylamino)phenyl-2,3-dihydro-3- hydroxychromen-4-one (1b)}

Yellow-brown solid, physical data is summarized in Table 3. IR (KBr, $\left.\mathbf{~ c m}^{-1}\right)$ : $3355(\mathrm{Ar}-\mathrm{OH}), 1556(\mathrm{Ar} \mathrm{C}=\mathrm{C}$ str), 1689 (C=5O str), 1327 (C-O str), 2895, (C-H str), 3027(Ar-H), 1318, (C-N). ${ }^{1} \mathbf{H}$ NMR(400MHz, DMSO $\delta$, TMS $=0): \delta=12.69(1 \mathrm{H}, \mathrm{s}, 3-\mathrm{OH}$, Exchangeable' with $\left.\mathrm{D}_{2} \mathrm{O}\right) .7 .59$. $(1 \mathrm{H}, \mathrm{d}, 5-\mathrm{H}, J=8.42 \mathrm{~Hz}), 7.51(1 \mathrm{H}$,dd, $7-\mathrm{H} J=8.24 \mathrm{~Hz}), 7.44\left(2 \mathrm{H}, \mathrm{m}, 2^{\prime}, 6^{\prime}-\mathrm{H}\right), 7.39(1 \mathrm{H}, \mathrm{dd}$, $6-\mathrm{H}, \mathrm{J}=7.76 \mathrm{~Hz}), 6.97(1 \mathrm{H}, \mathrm{d}, 8-\mathrm{H}, \mathrm{J}=8.23 \mathrm{~Hz}), 6.74$ $\left(2 \mathrm{H}, \mathrm{m}, 3{ }^{\prime}{ }^{\prime}-\mathrm{H}\right) 5.60(1 \mathrm{H}, \mathrm{d}, 3-\mathrm{H}, \mathrm{J}=12.56 \mathrm{~Hz}), 5.53$ $(1 \mathrm{H}, \mathrm{d}, 2-\mathrm{H}, \mathrm{J}=8.65 \mathrm{~Hz}), 2.97$ (6H, s, 4'-Dimethyl aimno), ${ }^{13} \mathrm{C}$ NMR (400 MHz, $\delta, \mathrm{CDCl3}$, TMS=0): Aromatic Ring-A (152.24, 132.12, 131. 87, 124.43, 122.49, 117.10) $178.88(\mathrm{C}=\mathrm{O}), 148.32$ (2-C), 138.35 (3-C), Aromatic Ring B (153.44, 147.89, 127.35, 127.36, 118.68, 118.68,) 40.16, 40.17, N-(CH3). TOF MS ES+ $(\mathrm{m} / \mathrm{z})=282$.

\section{2-(4-(chlorophenyl)-2,3-dihydro-3- hydroxychromen-4-one (2b)}

Yellow-white solid, physical data is summarized in Table 3. IR (KBr, $\mathbf{~ c m}^{-1}$ ): 3388 (Ar-OH), 1528 ( $\mathrm{Ar} \mathrm{C}=\mathrm{C}$ str), 1685 (C=O str), 1333 (C-O str), 3061 (Ar-H), 776 (C-Cl). ${ }^{1} \mathbf{H} \quad \mathbf{N M R}(400 \mathbf{M H z}, \quad$ DMSO $\quad \delta, \quad$ TMS $=0)$ : $\delta=12.16\left(1 \mathrm{H}, \mathrm{s}, 3-\mathrm{OH}\right.$, Exchangeable with $\left.\mathrm{D}_{2} \mathrm{O}\right)$,
7.62(1H,d, 5-H J= $8.00 \mathrm{~Hz}), 7.35(1 \mathrm{H}, \mathrm{dd}, 7-\mathrm{H}$, $\mathrm{J}=7.68 \mathrm{~Hz}), 7.25$ (2H, m, 2', (6'-H,), 7.19 (2H, m, 3', 5'-H, ), $7.03(1 \mathrm{H}, \mathrm{dd}, 6-\mathrm{H}, \mathrm{J}=3.05 \mathrm{~Hz}), 6.94(1 \mathrm{H}, \mathrm{d}, 8-\mathrm{H}, \mathrm{J}=8.04$ $\mathrm{Hz}), 5.60(1 \mathrm{H}, \mathrm{d}, 3-\mathrm{H}, \mathrm{J}=12.56 \mathrm{~Hz}), 5.53(1 \mathrm{H}, \mathrm{d}, 2-\mathrm{H}$, $\mathrm{J}=8.65 \mathrm{~Hz}),{ }^{13} \mathrm{C}$ NMR $(400 \mathrm{MHz}, \boldsymbol{\delta}, \mathbf{C D C l} 3$, $\mathbf{T M S}=\mathbf{0})$ : Aromatic Ring-A (153.23, 132.56, 131.81, 125.17, 121.49, 115.37), 187.49 (C=O), 153.46 (2-C), 145.59 (3-C), Aromatic Ring B (146.09, 138.79, 135.20, 135.91, 126.67, 126.37, TOF MS ES $+(\mathrm{m} / \mathrm{z})=275.5$.

\section{2-(furan-2-yl)-2,3-dihydro-3-hydroxychromen-4- one (3b)}

Yellow solid, physical data is summarized in Table 3. IR (KBr, $\left.\mathbf{~ c m}^{-1}\right): 3388(\mathrm{Ar}-\mathrm{OH}), 1527(\mathrm{Ar} \mathrm{C}=\mathrm{C}$ str), 1685 (C=O str), 1352 (C-O str), 3071 (Ar-H). ${ }^{1} \mathbf{H}$ NMR(400MHz, CMSO $\delta$, TMS $=0): \delta=11.90(1 \mathrm{H}, \mathrm{s}$, $3-\mathrm{OH}$, Exchangeable with $\left.\mathrm{D}_{2} \mathrm{O}\right) 7.69(1 \mathrm{H}, \mathrm{d}, 5-\mathrm{H}$ $J=8.20 \mathrm{~Hz}), 7.38(1 \mathrm{H}, \mathrm{dd}, 7-\mathrm{H}, \mathrm{J}=10.60 \mathrm{~Hz}), 7.20(1 \mathrm{H}$, $\mathrm{dd}, 6-\mathrm{H}, \mathrm{J}=4.04 \mathrm{~Hz}), \quad 7.09\left(1 \mathrm{H}, \mathrm{d}, 3^{\prime}-\mathrm{H}, \mathrm{J}=8.5 \mathrm{~Hz}\right)$, $6.99(1 \mathrm{H}, \mathrm{d}, 8-\mathrm{H}, \mathrm{J}=8.04 \mathrm{~Hz}), 6.77\left(1 \mathrm{H}, \mathrm{m}, 4^{\prime}-\mathrm{H}\right), 5.58(1 \mathrm{H}$, d, 3-H, J=12.56Hz), $5.52(1 \mathrm{H}, \mathrm{d}, 2-\mathrm{H}, \mathrm{J}=8.63 \mathrm{~Hz}),{ }^{13} \mathrm{C}$ NMR (400 MHz, $\delta, \mathbf{C D C 1 3}$, TMS=0): Aromatic Ring-A (152.85, 133.03, 130.31, 129.54, 119.58, 116.31), $187.38(\mathrm{C}=\mathrm{O}), 155.67$ (3-C), 140.59 (2-C), Aromatic Ring B (15.416, 142.68, 123.86, 112.73, ). TOF MS ES+ $(\mathrm{m} / \mathrm{z})=229.5$

\section{2-(4-(dimethylamino)phenyl-2,3-dihydro-3- hydroxy-7-methoxychromen-4-one (4b)}

Yellow-brown, physical data is summarized in Table 3. IR (KBr, cm $\left.{ }^{-1}\right): 3356$ (Ar-OH), 1570 (Ar C=C str), 1688 (C=O str), 1340 (C-O str), 2930,(H str), 3025 (Ar-H), 1247 (O-C), 1313, (C-N). ${ }^{1} \mathbf{H}$ NMR(400MHz, DMSO $\delta$, TMS $=0): \delta=12.00(1 \mathrm{H}, \mathrm{s}, 3-\mathrm{OH}$, Exchangeable 
with water), $7.57(1 \mathrm{H}, \mathrm{d}, 5-\mathrm{H}, J=8.00 \mathrm{~Hz}), 7.24(2 \mathrm{H}, \mathrm{m}$ ,2',6'-H ), $7.05(2 \mathrm{H}, \mathrm{m}, 3$ '5'-H), $6.98(1 \mathrm{H}, \mathrm{d}, 6-\mathrm{H}, \mathrm{J}=7.76$ $\mathrm{Hz}), 6.95(1 \mathrm{H}, \mathrm{s}, 8-\mathrm{H}), 5.58(1 \mathrm{H}, \mathrm{d}, 3-\mathrm{H}, \mathrm{J}=12.56 \mathrm{~Hz})$, $5.52(1 \mathrm{H}, \mathrm{d}, 2-\mathrm{H}, \mathrm{J}=8.63 \mathrm{~Hz}), 3.89\left(3 \mathrm{H}, \mathrm{s}, 7-\mathrm{OCH}_{3}\right), 2.06$ (6H, s,4'-Dimethyl aimno), ${ }^{13} \mathbf{C}$ NMR (400 MHz, $\delta, \mathbf{C D C l}$, TMS $=0)$ : Aromatic Ring-A $(157.77,152.34$, 132. 43, 122.12, 110.34, 108.30, 60.09) $188.73(\mathrm{C}=\mathrm{O})$, 153.46 (2-C), 144.78 (3-C), Aromatic Ring B (145.34, 134.75, 127.23, 127.33, 115.68, 115.50,) 40.01, 39.98, $\mathrm{N}-(\mathrm{CH} 3)_{2}$. TOF MS ES+ (m/z)=314

\section{2-(4-(chlorophenyl)-2,3-dihydro-3-hydroxy-7- methoxychromen-4-one (5b)}

Yellow-brown, physical data is summarized in Table 3. IR (KBr, $\left.\mathbf{~ c m}^{-1}\right)$ : 3356 (Ar-OH), 1527 (Ar C=C str), 1689 (C=O str), 1328(C-O str), 2939, (C-H str), 3083 (Ar-H), 1245(O-C), 777 (C-Cl). ${ }^{1} \mathbf{H}$ NMR(400 MHz, DMSO $\delta$, TMS $=0): \delta=), 12.11(1 \mathrm{H}, \mathrm{s}, 3-\mathrm{OH}$, Exchangeable with water), $7.68(1 \mathrm{H}, \mathrm{d}, 5-\mathrm{H} J=8.00 \mathrm{~Hz}), 7.35\left(2 \mathrm{H}, \mathrm{m}, 2^{\prime}, 6^{\prime}-\right.$ $\mathrm{H}$ ), $7.26\left(2 \mathrm{H}, \mathrm{m}, 3^{\prime}, 5^{\prime}-\mathrm{H},\right), 7.03(1 \mathrm{H}, \mathrm{d}, 6-\mathrm{H}, \mathrm{J}=8.04 \mathrm{~Hz})$, $6.98(1 \mathrm{H}, \mathrm{s}, 8-\mathrm{H}),, 5.58(1 \mathrm{H}, \mathrm{d}, 3-\mathrm{H}, \mathrm{J}=12.56 \mathrm{~Hz}), 5.52$ $(1 \mathrm{H}, \mathrm{d}, 2-\mathrm{H}, \mathrm{J}=8.63 \mathrm{~Hz}), 3.88\left(3 \mathrm{H}, \mathrm{s}, 7-7-\mathrm{OCH}_{3}\right)^{13} \mathbf{C} \mathbf{~ N M R}$ (400 MHz, $\delta, \mathbf{C D C l 3}$, TMS=0): Aromatic Ring-A (160.94, 153.82, 132.88, 127.89, 118.60, 117.13, 59.89), $188.38(\mathrm{C}=\mathrm{O}), 141.36$ (3-C), 155.59 (2-C), Aromatic Ring B (160.43, 145.67, 131.66, 131.56, 124.37, 124.29,). TOF MS ES $+(\mathrm{m} / \mathrm{z})=305$.

\section{2-(furan-2-yl)-2,3-dihydro-3-hydroxy-7- methoxychromen-4-one (6b)}

Yellow-brown, physical data is summarized in Table 3. IR (KBr, $\mathbf{c m}^{-1}$ ): 3365 (Ar-OH), 1521 (Ar C=C str), 1659 (C=O str), 1309 (C-O str), 2892, (C-H str), 3090 $(\mathrm{Ar}-\mathrm{H}), 7625$ (C-Cl). ${ }^{1} \mathbf{H} \mathbf{~ N M R}\left(400 \mathbf{~ M H z}, \delta, \mathbf{C D C l}_{3}\right.$, TMS=0): $8.06(1 \mathrm{H}, \mathrm{d}, 5-\mathrm{H}, \mathrm{J}=8.84 \mathrm{~Hz}), \quad 7.61(\mathrm{IH}, \mathrm{d}$, $3-\mathrm{H}, \mathrm{J}=8.0 \mathrm{~Hz}), 6.83(1 \mathrm{H}, \mathrm{d}, 6-\mathrm{H}, \mathrm{J}=8.60 \mathrm{~Hz}), 6.75(1 \mathrm{H}$, dd, 4'- $-\mathrm{H}, \mathrm{J}=3.00 \mathrm{~Hz}), 6.58(1 \mathrm{H}, \mathrm{s}, 8-\mathrm{H}), 5.88(1 \mathrm{H}, \mathrm{s}, 3-\mathrm{OH})$ Exchangeable with $\mathrm{D}_{2} \mathrm{O}, 5.68(1 \mathrm{H}, \mathrm{d}, 2-\mathrm{H}, \mathrm{J}=12.56 \mathrm{~Hz})$, $5.52(1 \mathrm{H}, \mathrm{d}, 3-\mathrm{H}, \mathrm{J}=8.63 \mathrm{~Hz}), 3.82\left(3 \mathrm{H}, \mathrm{s}, 7-\mathrm{OCH}_{3}\right),{ }^{13} \mathrm{C}$ NMR (400 MHz, $\delta, \mathbf{C D C 1 3}, \mathbf{T M S}=\mathbf{0})$ : Aromatic Ring-A (164.35, 151.23, 135.69, 119.36, 114.26, 108.70, 60.35), 188.87 (C=O), 135.33 (3-C), 145.47 (2-C), Aromatic Ring B (148.36, 103.09, 112.36, 111.16,).). TOF MS ES $+(\mathrm{m} / \mathrm{z})=261$.

\section{2-(4-(dimethylamino)phenyl-2,3-dihydro-3- hydroxy-6-methylchromen-4-one (7b)}

Yellow-brown, physical data is summarized in Table 3. IR (KBr, $\mathbf{~ c m}^{-1}$ ): 3405 (Ar-OH), 1533(Ar C=C str), 1690 (C=5O str), 1360 (C-O str), 2932, (C-H str), 3033 (Ar-H), 1319 (C-N) ${ }^{1} \mathbf{H}$ NMR(400MHz, DMSO $\delta$, TMS=0): $\delta=12.7\left(1 \mathrm{H}, \mathrm{s}, 3-\mathrm{OH}\right.$, Exchangeable with $\left.\left.\mathrm{D}_{2} \mathrm{O}\right),\right), 7.59$ $(1 \mathrm{H}, \mathrm{d}, 5-\mathrm{H}, J=8.40 \mathrm{~Hz}), 7.48\left(2 \mathrm{H}, \mathrm{m}, 2^{\prime}, 6^{\prime}-\mathrm{H}\right), 7.45$
$(1 \mathrm{H}, \mathrm{d}, 7-\mathrm{H}, \mathrm{J}=7.80 \mathrm{~Hz}), 6.94(1 \mathrm{H}, \mathrm{d}, 8-\mathrm{H}, \mathrm{J}=8.20 \mathrm{~Hz}$ ), $6.70\left(2 \mathrm{H}, \mathrm{m}, 3^{\prime} 5^{\prime}-\mathrm{H}\right), 5.68(1 \mathrm{H}, \mathrm{d}, 2-\mathrm{H}, \mathrm{J}=12.56 \mathrm{~Hz})$, $5.58(1 \mathrm{H}, \mathrm{d}, 3-\mathrm{H}, \mathrm{J}=8.63 \mathrm{~Hz}), \quad 3.08(6 \mathrm{H}, \mathrm{s}, 4$ '-Dimethyl aimno), $2.44\left(3 \mathrm{H}, \mathrm{s}, 6-\mathrm{CH}_{3}\right),{ }^{13} \mathrm{C}$ NMR (400 MHz, $\delta$, CDC13, TMS=0): Aromatic Ring-A (152.35, 151.38, 128.20, 122.34, 116.05, 110.47, 30.57) $187.31(\mathrm{C}=\mathrm{O})$, 153.46 (2-C), 147.78 (3-C), Aromatic Ring B (151.41, 143.46, 123.24, 123.23, 116.68, 116.90, ) 41.56, 41.49, $\mathrm{N}-(\mathrm{CH} 3)_{2}$. TOF MS ES+ $(\mathrm{m} / \mathrm{z})=298$.

\section{2-(4-(chlorophenyl)-2,3-dihydro-3-hydroxy-7- methylchromen-4-one (8b)}

Yellow solid, physical data is summarized in Table 3. IR (KBr, cm $\left.{ }^{-1}\right): 3395$ (Ar-OH), 1565 (Ar C=C str), 1688 (C=O str), 1324 (C-O str), 2960, (C-H str), 3070 $(\mathrm{Ar}-\mathrm{H}), 776$ (C-Cl). ${ }^{1} \mathbf{H} \mathbf{N M R}\left(400 \mathbf{M H z}, \delta, \mathbf{C D C l}_{3}\right.$, TMS $=0): \delta=12.09(1 \mathrm{H}, \mathrm{s}, 3-\mathrm{OH}$, Exchangeable with $\left.\mathrm{D}_{2} \mathrm{O}\right), 7.65(\mathrm{IH}, \mathrm{d}, 5-\mathrm{H}, \mathrm{J}=8.04 \mathrm{~Hz}), 7.31\left(2 \mathrm{H}, \mathrm{m} .2^{\prime}, 6^{\prime}-\mathrm{H}\right)$, $7.18\left(2 \mathrm{H}, \mathrm{m}, 3^{\prime}, 5\right.$ '-H), $6.92(1 \mathrm{H}, \mathrm{d}, 7-\mathrm{H}, \mathrm{J}=8.00 \mathrm{~Hz}), 6.05$ $(1 \mathrm{H}, \mathrm{d}, 8-\mathrm{H}, \mathrm{J}=8.02 \mathrm{~Hz}), 5.68(1 \mathrm{H}, \mathrm{d}, 2-\mathrm{H}, \mathrm{J}=12.56 \mathrm{~Hz})$, $5.58(1 \mathrm{H}, \mathrm{d}, 3-\mathrm{H}, \mathrm{J}=8.63 \mathrm{~Hz}), 2.64\left(3 \mathrm{H}, \mathrm{s}, 6-\mathrm{CH}_{3}\right),{ }^{13} \mathrm{C}$ NMR (400 MHz, $\delta, \mathbf{C D C l 3}$, TMS $=0)$ ): Aromatic Ring-A (154.09, 133.24, 131.37, 130.77, 126.31, 116.34, 108.47, 26.78), 188.44 (C=O), 144.67 (3-C), 148.34 (2-C), Aromatic Ring B (151.09, 148.05, 127.28, 127.16, 117.24, 117.34,). TOF MS ES+ (m/z)= 287.

\section{2-(furan-2-yl)-2,3-dihydro-3-hydroxy-6- methylchromen-4-one (9b)}

Yellow-brown, physical data is summarized in Table 3. IR (KBr, cm $\left.{ }^{-1}\right): 3355$ (Ar-OH), 1528 (Ar C=C str), 1690 (C=O str), 1332 (C-O str), 2937 (C-Hstr), 3082 $(\mathrm{Ar}-\mathrm{H}) .{ }^{1} \mathbf{H} \quad \mathbf{N M R}(400 \mathrm{MHz}, \quad \mathbf{C M S O} \quad \delta, \quad \mathrm{TMS}=0)$ : $\delta=11.98\left(1 \mathrm{H}, \mathrm{s}, 3-\mathrm{OH}\right.$, Exchangeable with $\left.\mathrm{D}_{2} \mathrm{O}\right)$, $7.71(1 \mathrm{H}, \mathrm{s}, 6$ ' $-\mathrm{H}), 7.40(1 \mathrm{H}, \mathrm{d}, 7-\mathrm{H}, \mathrm{J}=8.50 \mathrm{~Hz}), 7.20$ $\left(1 \mathrm{H}, \mathrm{d}, 3^{\prime}-\mathrm{H}, \mathrm{J}=8.0 \mathrm{~Hz}\right), 6.87(1 \mathrm{H}, \mathrm{d}, 8-\mathrm{H}, \mathrm{J}=8.01 \mathrm{~Hz})$, $6.76\left(1 \mathrm{H}, \mathrm{m}, 4^{\prime}-\mathrm{H}\right), 5.68(1 \mathrm{H}, \mathrm{d}, 2-\mathrm{H}, \mathrm{J}=12.56 \mathrm{~Hz}), 5.58$ $(1 \mathrm{H}, \mathrm{d}, 3-\mathrm{H}, \mathrm{J}=8.63 \mathrm{~Hz}), 2.48\left(3 \mathrm{H}, \mathrm{s}, 6-\mathrm{CH}_{3}\right),{ }^{13} \mathbf{C} \mathbf{~ N M R}$ (400 MHz, $\delta, \mathrm{CDCl}$, TMS=0): Aromatic Ring-A (162.45, 140.36, 132.46, 131.62, 125.27, 116.58, 25.02), 188.87 (C=O), 149.54 (3-C), 143.80 (2-C), Aromatic Ring B (153.09, 145.80, 112.98, 112.790, ). TOF MS $\mathrm{ES}+(\mathrm{m} / \mathrm{z})=245$.

\section{2,3-dihydro-3-hydroxy-2-(4-nitrophenyl)chromen- 4-one (10b)}

Yellow-brown, physical data is summarized in Table 3. IR (KBr, $\left.\mathbf{~ c m}^{-1}\right): 3350$ (Ar-OH), 1552 (Ar C=C str), 1688 (C=5O str), 1325 (C-O str), 2892, (C-H str), 3025(Ar$\mathrm{H}), \quad 1317, \quad(\mathrm{C}-\mathrm{N}) .{ }^{1} \mathbf{H} \quad \mathbf{N M R}(400 \mathrm{MHz}, \quad$ DMSO $\delta$, TMS $=0): \delta=12.69(1 \mathrm{H}, \mathrm{s}, 3-\mathrm{OH})$, Exchangeable with $\mathrm{D}_{2} \mathrm{O}, 7.59 .\left(2 \mathrm{H}, \mathrm{m}, 3^{\prime} 5,-\mathrm{H},\right), 7.51(1 \mathrm{H}, \mathrm{d}, 5-\mathrm{H} J=8.24 \mathrm{~Hz})$, $7.44\left(2 \mathrm{H}, \mathrm{m}, 2^{\prime}, 6^{\prime}-\mathrm{H}\right), 7.39(1 \mathrm{H}, \mathrm{dd}, 7-\mathrm{H}, \mathrm{J}=7.76 \mathrm{~Hz})$, 
$6.97(1 \mathrm{H}, \mathrm{d}, 6-\mathrm{H}, \mathrm{J}=8.23 \mathrm{~Hz}), 6.74(1 \mathrm{H}, \mathrm{d}, 8-\mathrm{H}, \mathrm{J}=7.50$ $\mathrm{Hz}), 5.68(1 \mathrm{H}, \mathrm{d}, 2-\mathrm{H}, \mathrm{J}=12.56 \mathrm{~Hz}), 5.58(1 \mathrm{H}, \mathrm{d}, 3-\mathrm{H}$, $\mathrm{J}=8.63 \mathrm{~Hz}),{ }^{13} \mathbf{C}$ NMR $(400 \mathrm{MHz}, \delta, \mathbf{C D C l} 3$, TMS $=\mathbf{0})$ : Aromatic Ring-A (152.24, 132.12, 131. 87, 124.43, 122.49, 117.10) $178.88(\mathrm{C}=\mathrm{O}), 148.32$ (2-C), 138.35 (3-C), Aromatic Ring B (153.44, 147.89, 127.35, 127.36, $118.68,118.68$, , 40.16, 40.17, $\left(\mathrm{C}-\mathrm{NO}_{2}\right)$. TOF MS ES+ $(\mathrm{m} / \mathrm{z})=282$.

\section{Physicochemical characterization of 3-methylflavanone analogues (1a-10a)}

Physicochemical characterizations of 3-methylflavanone analogues are listed in Table 2.

\section{Physicochemical characterization of 3-hydroxyflavanone analogues (1b-10b)}

Physicochemical characterization of 3-hydroxyflavanone analogues are listed in Table 3.

\section{Anti-leishmanial Activity}

The anti-leishmanial activities of synthesized compounds were screened against promastigotes of L.donovani. Amphotericin B and Sodium Stibugluconate were used as standard drugs and exhibited $100 \%$ inhibition. The results are presented in Table 4.

\section{Docking Study}

All the twenty compounds were docked into the active site of the PTR1 and the estimated free binding energy were presented in Table 5 and the interaction of most promising test compounds with receptors were illustrated in Figure $2 \mathrm{~A}$ and $2 \mathrm{~B}$ respectively.

\section{DISCUSSION}

All the selected 20 compounds were investigated for their activities against axenic L. donovani prmastigotes, grown in simple media have been used as test parasite to screen potential antileishmanial agents and the simplicity of this system accounts for its wide popularity, and the results were expressed as \% inhibition illustrated in Table 4 with few exceptions, all 3- methyl and 3-hdroxy flavanone showed significant antileishmanial activity. The test compounds such as 4b (95\%), 10b (93\%), 5b $(92 \%), 6 \mathrm{~b}(91 \%)$, and $3 \mathrm{a}(90 \%)$ being the most potent. Their $\%$ inhibitions were almost comparable to that of Amphotericin B and Sodium Stibugluconate the antileishmanial drug used in the clinic. Starting with the flavanone, the insertion of a single $\mathrm{OH}$ group at the benzo- $\gamma$ - chromone (at-3-position) portion of the flavone structure have a notable influence, but insertion of one more $\mathrm{OH} / \mathrm{OCH}_{3}$ on ring $\mathrm{A}$ and $\mathrm{B}$ functions significantly enhanced the leishmanicidal potential. Particularly important positions were C-5, C-7 on ring

\section{Table 4: Antileishmanial Activity (MTT Assay)}

\begin{tabular}{|c|c|}
\hline Compound & $\begin{array}{c}\text { \%Inhibition of L. donovani } \\
\text { promastigotes 1a12 }\end{array}$ \\
\hline $2 a$ & 15 \\
\hline $3 a$ & 90 \\
\hline $4 a$ & 84 \\
\hline $5 a$ & 82 \\
\hline $6 a$ & 18 \\
\hline $7 a$ & 80 \\
\hline $8 a$ & 71 \\
\hline $9 a$ & 81 \\
\hline $10 a$ & 68 \\
\hline $1 b$ & 80 \\
\hline $2 b$ & 62 \\
\hline $3 b$ & 81 \\
\hline $4 b$ & 95 \\
\hline $5 b$ & 92 \\
\hline $6 b$ & 91 \\
\hline $7 b$ & 78 \\
\hline $8 b$ & 72 \\
\hline $9 b$ & 87 \\
\hline $10 b$ & 93 \\
\hline & \\
\hline
\end{tabular}

$\%$ Inhibition of of $L$. donovani promastigotes at $100 \mu \mathrm{g} / \mathrm{ml}$ of each test compound. Each data value represents mean \pm SEM of at least three experiments performed in duplicate. $100 \%$ inhibition was observed by standard drug at 100

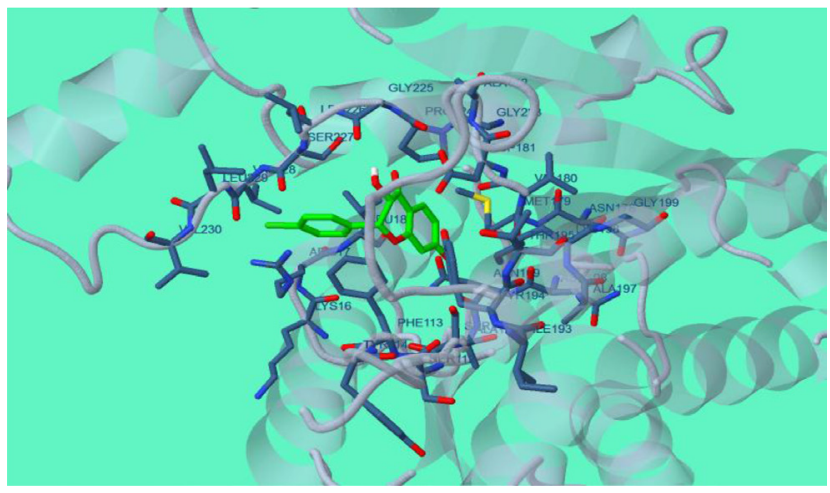

Figure 2A: Stereoview of the complex formed by PTR1 and the docked compound (4b).The amino acids Arg 17, Lys 199, Asn109, Asp 181 and Phe113 were involved in interaction with compounds.

A. Hydroxylation on ring B had some impact on the activity, but a clear SAR could not be observed. Further, compounds such as 9b (87\%), 4a (84\%), 5a (82\%), 9a (81\%), 3b (81\%), $7 \mathrm{a}(80 \%)$ and $1 \mathrm{~b}(80 \%)$ have shown appreciable leishmanicidal activity as compared with that of the standard drugs, as most of the test compounds were in conjugation with hydroxyl or electron donating groups except nitro group. However, compounds such as $8 \mathrm{~b}(72 \%), 8 \mathrm{a}(71 \%), 10 \mathrm{a}(68 \%)$ and $2 \mathrm{~b}(62 \%)$ exhibited moderate activity against promastigates of L.donovani, as these compounds were having either mild 


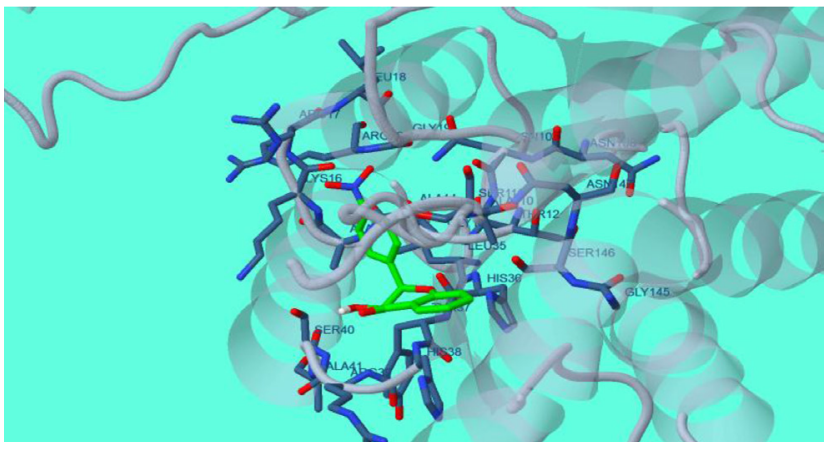

Figure 2B: Stereoview of the complex formed by PTR1 and the docked compound (10b). The amino acids Lys 16, Ser 40, Asn 140, and Arg 17 were involved in interaction with compounds.

electron withdrawing group or without electronic effect on main nucleus. The presence of strong electron withdrawing group on the entire structure significantly reduced the leishmanicidal activity as observed in compounds, such as $1 \mathrm{a}(12 \%) 2 \mathrm{a}(15 \%)$, $6 \mathrm{a}(18 \%)$ see Table 1. Basedontheinformationobtained, itisdifficulttodecipher empirical SARs among the test compounds investigated in the current study. It is quite noteworthy in the finding that majority of leishmanicidal compounds have a typical 3-hydroxy flavanone structure with one or more substitution by hydroxyl or methoxy groups or electron donating group(s). Furthermore, drug receptor interaction was carried out to validate our findings. The ligands were ranked according to docking score / estimated free energy of binding. The free energy of binding of ligands was in the range between -3.15 to $-7.84 . \mathrm{Kcal} / \mathrm{mole}$. Top ranked compound (4b) and (10b) with -7.84 and$7.77 \mathrm{Kcal} / \mathrm{mole}$ free energy of binding, respectively, were in correlation with wet lab experiments. The protein ligand analysis also has shown its strong interactions with target protein and had five hydrogen bond interaction in (4b) and four hydrogen bond interaction in (10b). The residues involved in hydrogen bond interaction were Arg 17, Lys 199, Asn109, Asp 181 and Phe113 in (4b) (Figure 2A) and Lys 16, Ser 40, Asn 140, and Arg 17 in (10b) (Figure 2B) with the active site of PTR1. Virtual screening of 100 3-substituted flavanone analogues library resulted in the identification of 20 compounds. Out of 100 compounds, 20 compounds with the lowest estimated free energy of binding were selected for synthesis. Among synthesized compounds top ranked compounds (4b) and (10b) according to estimated free energy of binding (-7.84 and-7.77 Kcal/mole respectively), also had promising anti-leishmanial activity in wet lab experiments. The excellent interactions of PTR1 with all five top ranked compounds (4b), (10b),

\begin{tabular}{|c|c|}
\hline $\begin{array}{c}\text { Table 5: Estimated free energy of binding of isolated } \\
\text { compounds in the target PTR1. }\end{array}$ \\
\hline Compounds & $\begin{array}{c}\text { Estimated free energy of binding } \\
\text { (Kcal/mol) }\end{array}$ \\
\hline $4 b$ & -7.84 \\
\hline $10 b$ & -7.77 \\
\hline $5 b$ & -7.76 \\
\hline $6 b$ & -7.62 \\
\hline $3 a$ & -7.56 \\
\hline $9 b$ & -6.95 \\
\hline $4 a$ & -6.51 \\
\hline $5 a$ & -6.40 \\
\hline $9 a$ & -6.16 \\
\hline $3 b$ & -6.01 \\
\hline $7 b$ & -5.73 \\
\hline $1 b$ & -5.61 \\
\hline $8 b$ & -5.54 \\
\hline $8 a$ & -4.78 \\
\hline $2 b$ & -4.06 \\
\hline $1 a$ & -3.66 \\
\hline $2 a$ & -3.52 \\
\hline $6 a$ & -3.39 \\
\hline $7 a$ & -3.21 \\
\hline $10 a$ & -3.15 \\
\hline
\end{tabular}

${ }_{3}$ D structures of PTR1, (from $2 X O X$ ), was used for virtual screening.

(5b), (6b) and (3a) indicated a high degree of coherent relationship between in silico approach and in vitro studies. An in-vitro promising anti-leishmanial activity of the compounds demands further in-vivo and clinical studies and these compounds might find an important place in the new array of molecules targeting PTR1 dependent biological functions as anti-leishmanial agents. However, there are some limitations of using promastigotes for in vitro anti-leishmanial study; the metabolism and ecology of promastigote differ so widely from those of amastigote that screening data obtained from in vitro test with promastigote have very little value in animals. ${ }^{29,30}$ Another condition which reduces leishmanicidal action in vitro is lower temperature $\left(24^{\circ} \mathrm{C}\right)$ at which the culture normally grows, as opposed to the in vivo temperature of $37^{\circ} \mathrm{C}$. The promastigote in culture at $37^{\circ} \mathrm{C}$ will survive but not multiply. Further, the promastigote culture represents an artificial situation and is not always showing a significant value for drug screening. ${ }^{31}$

\section{CONCLUSION}

This study reports on the in vitro anti-leishmanial activity of a number of flavanone analogues synthesized from 
2'-hydroxypropiohenones, 2'-hydroxyacetophenone, and substituted benzaldehyde. The study was prompted by the need of novel, efficacious and cost-effective medicines against this parasitic disease. After elucidating the chemical structure of the newly synthesized flavanone analogues, they were evaluated for their potential leishmanicidal activity against cultured $L$. (L.) donovani promastigotes. These experiments revealed promising anti-leishmanial activity of particularly compound 10b, which also showed the best docking into the active site of pteridine reductase.

\section{ACKNOWLEDGEMENT}

The authors thank to Narayan Institute of Pharmacy to facilitate part of the research work. We must thank to SAIF, Auvtar Singh and Manish Kumar, Panjab University, Chandigarh, (India) to get spectral data of 1HNMR, C13NMR, and Mass Spectrometry.

\section{CONFLICTS OF INTEREST}

The authors declare no conflict of interest.

\section{ABBREVIATIONS USED}

PTR1: Pteridine Reductase; DHFR: Dihydrofolate reductase; SARs: Structure Activity Relationships DMSO: Dimethyl Sulphoxide; TLC: Thin Layer Chromatography; FBS: Fetal Bovine Serum; ELISA: Enzyme Linked Immunosorbent Assay; MTT: [3-(4.5-dimethylthiazol-2-yl)-2.5-diphenyl tetrazolium; TMS: Tetra Methyl Silane.

\section{REFERENCES}

1. Desjeux P. Leishmaniasis: current situation and new perspectives. Comp Immunol Microbiol Infect Dis. 2004;27:305-18.

2. Mahomoodally MF, Gurib-Fakim A, Subratty AH. Antimicrobial activities and phytochemical profiles of endemic medicinal plants of Mauritius. Pharm Biology. 2008;43(3):237-42.

3. Pandey AK. Anti-staphylococcal activity of a pan-tropical aggressive and obnoxious weed Parthenium histerophorus: an in vitro study. Nation Acad Sci Lett. 2007;30(11-12):383-6

4. Kelly E H, Anthony RT, Dennis JB. Flavonoid antioxidants:chemistry, metabolism and structure-activity relationships. J Nutr Biochem. 2002;13(10):572-84.

5. Choi JS, Chung HY, Kang SS, Jung MJ, Kim JW, No JK, et al. The structureactivity relationship of flavonoids as scavengers of peroxynitrite. Phytother Res. 2002;16(3):232-5.

6. Cos P, Ying L, Calomme M, Hu JP, Cimanga K, Van Poel B, et al. Structureactivity relationship and classification of flavonoids as inhibitors of xanthine oxidase and superoxide scavengers. J Nat Prod. 1998;61(1):71-6.

7. Yang JG, Liu BG, Liang GZ, Ning ZX. Structure-activity relationship of flavonoids active against lard oil oxidation based on quantum chemical analysis. Molecules. 2009;14(1):46-52.

8. Farkas O, Jakus J, Heberger K. Quantitative structure-antioxidant activity relationships of flavonoid compounds. Molecules. 2004;9(12):1079-88.
9. Cheong H, Ryu SY, Oak MH, Cheon SH, Yoo GS, Kim KM. Studies of structure activity relationship of flavonoids for the anti-allergic actions. Arch Pharm Res. 1998;21(4):478-80.

10. Nielsen SF, Boesen T, Larsen M, Schønning K, Kromann H. Antibacterial Chalcones bioisosteric replacement of the 4'-hydroxy group. Bioorgan Med Chem. 2004;12(11):3047-54.

11. Heim KE, Tagliaferro AR, Bobilya DJ. Flavonoid antioxidants: chemistry, metabolism and structure-activity relationships. J Nutr Biochem. 2002;13(10):572-84.

12. Hsieh HK, Lee TH, Wang JP, Wang JJ, Lin CN. Synthesis and antiinflammatory effect of chalcones and related compounds. Pharm Res. 1998;15(1):39-46.

13. Li R, Kenyon GL, Cohen FE, Chen X, Gong B, Dominguez JN, et al. In vitro antimalarial activity of chalcones and their derivatives. J Med Chem. 1995;38(26):5031-7.

14. Hardy LW, Matthews W, Nare B, Beverley SM. Biochemical and genetic tests for inhibitors of Leishmania pteridine pathways. Exp Parasitol. 1997;87(3):157-69.

15. Singh N, Mishra BB, Bajpai S, Singh RK, Tiwari VK. Natural product based leads to fight against leishmaniasis. Bioorg Med Chem. 2014;22(1):18-45.

16. Liu M, Wilairat P, Croft SL, Tan AL, Go ML. Structure-activity relationships of anti-leishmanial and antimalarial Chalcones. Bioorg Med Chem. 2003;11(13):2729-38.

17. Cushnie T, Lamb AJ. Antimicrobial activity of flavonoids. Int $\mathrm{J}$ Antimicrob Agents. 2005;26:343-56.

18. Hendrich AB. Flavonoid-membrane interactions: possible consequences for biological effects of some polyphenolic compounds. Acta Pharmacol Sin. 2006;27(1):27-40.

19. Jayashree BS, Alam A, Nayak Y, Vijay KD. Synthesis of 3-methylflavones and their antioxidant and antibacterial activities. Med Chem Res. 2012;21(8):1991-6.

20. Thakar GP, Janaki N, Rao BS. Reductions with diborane+ sodium borohydride in presence of lewis acids Benzopyrones. Indian J Chem. 1965;3(2):74.

21. Ouchi A, Sakai H, Oishi T, Kaneda M, Suzuki T, Saruwatari A. Photochemical reduction of flavone with $\mathrm{NaBH}_{4}$ in batch and micro-channel reactors using excimer lasers. J Photochem Photobiol A Chem. 2008;199(2-3):261-6.

22. Kamlesh N, Shivakumar T, Afroze Alam. Cytotoxicity and Anti-inflammatory Activity of Flavonoid Derivatives Targeting NF-kappaB. Recent Pat Inflamm Allergy Drug Discov. 2016;10(2):119-32.

23. Camacho M, Phillipson JD, Croft SL, Marley D, Kirby GC, Warhurst DC. Assessment of the antiprotozoal activity of Galphimia glauca and the isolation of new nor-secofriedelanes and nor-friedelanes. J Nat Prod. 2002;65(10):1457-61.

24. Grael CF, Albuquerque S, Lopes JL. Chemical constituents of Lychnophora pohlii and trypanocidal activity of crude plant extracts and of isolated compounds. Fitoterapia. 2005;76(1):73-82.

25. Jordão CO, Vichnewski W, de Souza GE, Albuquerque S, Lopes JL. Trypanocidal activity of chemical constituents of Lychnophora salicifolia. Mart Phytother Res. 2004;18(4):332-4.

26. Borgatti M, Chilin A, Piccagli L, Lampronti I, Bianchi N, Mancini I, et al. Development of a novel furocoumarin derivative inhibiting NF-KB dependent biological functions: Design, synthesis and biological effects. Eur J Med Chem. 2001;46(10):4870-7.

27. Piccagli L, Borgatti M, Nicolis E, Bianchi N, Mancini I, Lampronti I, et al. Virtual screening against nuclear factor $\mathrm{KB}(\mathrm{NF}-\mathrm{KB})$ of a focus library: Identification of bioactive furocoumarin derivatives inhibiting NF-KB dependent biological functions involved in cystic fibrosis. Bioorgan Med Chem. 2010;18(23): 8341-9.

28. Cosconati S, Forli S, Perryman AL, Harris R, Goodsell DS, Olson AJ. Virtual screening with AutoDock: theory and practice. Expert Opin Drug Discov. 2010;5(6):597-607.

29. Croft SL, Seifert K, Yardley V. Current scenario of drug development for 1eishmaniasis. Indian J Med Res. 2006;123(3):399-410.

30. Sunduru N, Nishi, Palne S, Chauhan PMS, Gupta S. Synthesis and antileishmanial activity of novel 2,4,6-trisubstituted pyrimidines and 1,3,5-triazines. Europ J Med Chem. 2009;44(6):2473-81.

31. Suman GN. Visceral leishmaniasis: experimental models for drug discovery. Indian J Med Res. 2011;133(1):27. 


\section{PICTORIAL ABSTRACT}

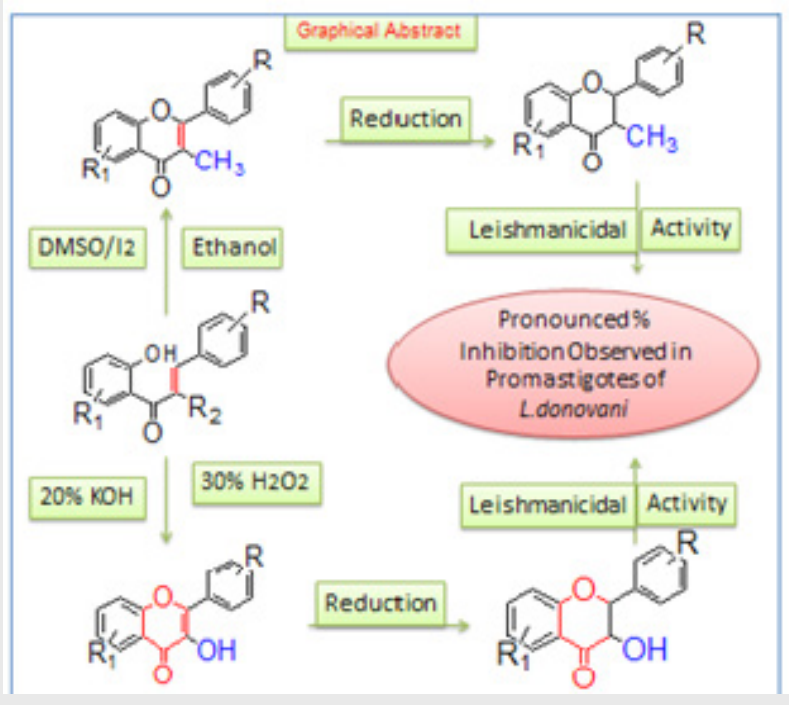

\section{About Authors}

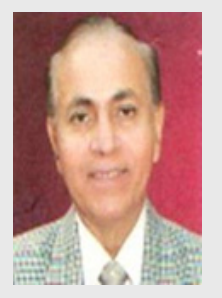

Mohan Lal Verma is Principal and Director cum Technical Advisor in Narayan Medical College \& Hospital, Jamuhar 821305, Sasaram Bihar. Earlier he served as Principal in Patna Medical College \& Hospital as well as in Nalanda Medical College \& Hospital He has done MBBS, MD in Pharmacology, He has been awarded so many titles in the field of Medical Science.. His work interest is multi-disciplinary research focus on development of new drugs related to leihmaniasis. He has a huge experience to handle academic and administrative profile of the colleges and University. Moreover, Dr. M.L. Verma published 6 books and more than 100 research and review papers in peered reviewed journals. He is chairman of the various committees or advisory board of many government Universities.

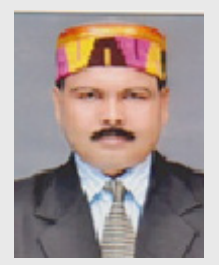

Afroze Alam is specialized in Pharmaceutical Chemistry at M. Pharma level and did his Ph.D. in Pharmaceutical/Medicinal and Phytochemistry. He is working as Associate professor at Narayan Institute of Pharmacy, Jamuhar, Sasaram-821305, Rohtas Bihar, India. His main area of work is drug design and the natural product chemistry of anticancer, antibacterial, antiIsieshmanial, antimalarial and anti-diabetic molecules. Moreover, his research interest includes nanobiomedicines. He published more than 30 papers in internationally recognized high impact biomedical and chemistry journals.

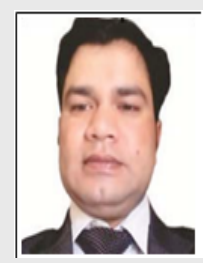

Mr. Kamlesh Naik did his post-graduation (2007) in Pharmaceutical Sciences from Nandha College of Pharmacy Erode, Chennai, . Presently, he is working as a Ph.D. Research fellow at Pharmaceutical research lab, Department of Pharmaceutical Chemistry, Nandha College of Pharmacy Erode, Tamil Nadu, India. Her research work has focus predominantly on the flavonoids nucleus and its associated pharmacological activities such as anti-oxidant, hepatoprotective, antiinflammatory and cytotoxic activity. He is going to face defense or Viva -Voce of his PhD work in April, 2018. He published 12 research and review article in reputed journals. 


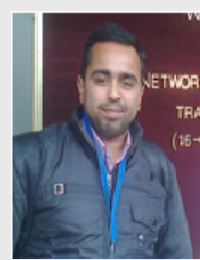

Mr. Mahfoozur-Rahman is currently working as a Pharmaceutics faculty at Department of Pharmaceutical Sciences, Faculty of Health Sciences, Sam Higginbottom Institute of Agriculture, Technology and Sciences (SHIATS), Allahabad, INDIA, Uttar Pradesh, India. He is a professional Pharmacy graduate; did his M.Pharm and pursuing Ph.D. in Pharmaceutical Science (specilization Pharmaceutics). He was awarded with senior fellowship from Council of Scientific and Industrial Research (CSIR), India. His research interests involve drug delivery and targeting, nanoparticulates and vesicular systems, nanotoxicology and pharmaceutical analysis. Mr. Mahfoozur-Rahman has published more than 50 papers including book chapters in peer-reviewed journals of high impact.

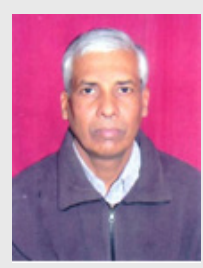

Shailendra Kumar did his M.Pharmacy from Manipal College of Pharmaceutical Sciences, Udupi Karnataka, India, He has been awarded PhD from Magadh University, Bodh Gaya, Presently he is working as Principal, Govt. Pharmacy Institute, Agam Kuan Patna Bihar-India. He is the members of many government regulatory bodies. He is specialized in Pharmaceutical Chemistry. His research area is profoundly related to the isolation of bioactive molecules from plant sources. He greatly contributes to the development of Pharmacy institute in Bihar. He published more than 15 papers in reputed journal with high impact.

Cite this article: Alam A, Pandit V, Kumar S, Naik KK, Rahman M, Verma ML. Anti-Leishmanial Activity of Flavanone Analogues Targeting Pteridine Reductase. Indian J of Pharmaceutical Education and Research. 2018;52(3)480-91. 